\title{
Type material of Acanthocephala, Nematoda and other non-helminths phyla (Cnidaria, Annelida, and Arthropoda) housed in the Helminthological Collection of the Oswaldo Cruz Institute/ FIOCRUZ (CHIOC), Rio de Janeiro, Brazil, from 1979 to 2016
}

\author{
Daniela A. Lopes', Delir Corrêa Gomes', Marcelo Knoff' \\ I Laboratório de Helmintos Parasitos de Vertebrados, Instituto Oswaldo Cruz, FIOCRUZ, Av. Brasil, 4365 \\ Rio de Janeiro, RJ, Brazil \\ Corresponding author: Marcelo Knoff (knoffm@ioc.fiocruz.br)
}

Academic editor: Fredric Govedich | Received 28 June 2017 | Accepted 8 August 2017 | Published 23 October 2017

http://zoobank.org/D94E8B43-C7A7-4473-86D4-FFBFAD6852DC

Citation: Lopes DA, Gomes DC, Knoff M (2017) Type material of Acanthocephala, Nematoda and other nonhelminths phyla (Cnidaria, Annelida, and Arthropoda) housed in the Helminthological Collection of the Oswaldo Cruz Institute/ FIOCRUZ (CHIOC), Rio de Janeiro, Brazil, from 1979 to 2016. ZooKeys 711: 1-52. https://doi. org/10.3897/zookeys.711.14753

\begin{abstract}
The third part of the catalogue of type material in the Helminthological Collection of the Oswaldo Cruz Institute/FIOCRUZ (CHIOC), comprising types deposited between 1979 and 2016, is presented to complement the first list of all types that was published in 1979. This part encompasses Acanthocephala, Nematoda and the other non-helminth phyla Cnidaria, Annelida, and Arthropoda. Platyhelminthes was covered in the first (Monogenoidea) and second (Rhabditophora Trematoda and Cestoda) parts of the catalogue published in September 2016 and March 2017, respectively. The present catalogue comprises type material for 116 species distributed across five phyla, nine classes, 50 families, and 80 genera. Specific names are listed systematically, followed by type host, infection site, type locality, and specimens with their collection numbers and references. Species classification and nomenclature are updated.
\end{abstract}

Copyright Daniela A. Lopes et al. This is an open access article distributed under the terms of the Creative Commons Attribution License (CC BY 4.0), which permits unrestricted use, distribution, and reproduction in any medium, provided the original author and source are credited. 


\section{Keywords}

Acanthocephalans, annelids, catalogue, copepods, holotype, myxozoans, nematodes, parasites, paratype

\section{Introduction}

The century-old Helminthological Collection of the Oswaldo Cruz Institute/FIOCRUZ (CHIOC), Rio de Janeiro, Brazil, contains helminths that form part of the fauna of Brazil, and other countries, and are from a wide range of hosts captured in a variety of different biomes. The samples are holotypes, paratypes, and representative specimens of Platyhelminthes, Acanthocephala, Nematoda, and other nonhelminth phyla. The CHIOC holds around 38,400 samples of helminth parasites from South America and other continents, and represents the largest such collection in Latin America and one of the largest collections worldwide (Rego 1982, Knoff et al. 2010). Details about the history and composition of CHIOC were presented in Lopes et al. (2016).

The first catalogue of all type material held in the CHIOC recorded 719 types of helminths (only holotypes or type series): 408 nematodes, 216 digenetic trematodes, 11 monogenoids, 52 acanthocephalans, 28 cestodes, and four of pentastomids (Rego et al. 1979). Since its publication, the collection has grown substantially with the number of types increasing significantly as well. Recently, Lopes et al. (2016, 2017) published another catalogue listing 203 type species of Monogenoidea and 104 type species of the other three classes of platyhelminths (Rhabditophora, Trematoda, and Cestoda). Thus, the present catalogue is the third list of type species held in this collection, and encompasses those of Acanthocephala, Nematoda, and the non-helminth phyla Cnidaria, Annelida and Arthropoda that have been deposited in CHIOC since 1979. The purpose of this article is to inform the scientific community about the types deposited in CHIOC as of December 1, 2016, and follows the articles of the International Code of Zoological Nomenclature (ICZN 1999).

\section{Materials and methods}

The specimens are stored in glass or plastic vials in $70 \%$ ethanol (with or without $5 \%$ glycerin), $4-10 \%$ formaldehyde (with or without $2 \%$ acetic acid), $70 \%$ ethanolformaldehyde-acetic acid (AFA) or as microscope slide preparations. All the material is available for consultation, but holotypes are not loaned. Unless otherwise stated, all type material is in good condition.

The catalogue is arranged taxonomically as phyla, classes, orders, families, genera, and species, under the original spelling and combinations. Phyla and classes are arranged phylogenetically, starting with helminth phyla. Orders, families, genera, and 
species are arranged alphabetically. The information on each entry is presented in the following format:

1. Original genus-species combination with author(s) and year of publication. An asterisk $\left(^{*}\right)$ denotes the type species of the genus.

2. Type host: updated scientific name, author(s) and year, with original scientific name in square brackets (when changed), followed by principal taxonomic group in parentheses.

3. Infection site in the host.

4. Type locality: country, province or state, department, specific locality and coordinates (if available).

5. Primary type status: sex (if applicable/ possible), CHIOC catalog number. Categories for types follow articles 73-75 of the Code (ICZN 1999).

6. Remark sections are inserted when necessary and include additional information about host, locality, or status of the types.

7. References include publications in which the species was described and those that mention type specimens in the CHIOC.

The valid names adopted for parasitized hosts follow specific bibliographies. Diplopod names are in accordance with Pérez-Asso (1996); beetles with Boucher (2005); crustaceans with Young (1998); fishes with Froese and Pauly (2016); amphibians with Frost (2016); reptiles with Uetz et al. (2016); birds with Lepage and Warnier (2014); and mammals with Wilson and Reeder (2005). Mention of acanthocephalans, nematodes, myxozoans, annelids, copepods, and host species in this list does not imply that the authors of the present report agree with their validity or taxonomy. Some species catalogued have been synonymized and the comments about their taxonomy are provided in the remark sections. The higher classification of Acanthocephala follows Amin (1985, 2013); Nematoda follows De Ley and Blaxter (2002, 2004); Myxozoa follows Fiala et al. (2015); Annelida follows Beesley et al. (2000) and Sawyer (1986); and Copepoda follows Martin and Davis (2001). The classification of families and genera follows specific references.

\section{Abbreviation list}

BMNH

CHFC

CHIBB

CHIOC
British Museum of Natural History, Collection at the Department of Zoology, Natural History Museum, London, England; Helminthological Collection of the Science College, University of the Republic, Montevideo, Uruguay;

Reference Helminthological Collection of the Parasitology Department, Bioscience Institute, Paulista State University (UNESP), Botucatu, São Paulo, Brazil;

Helminthological Collection of the Oswaldo Cruz Institute, FIOCRUZ, Rio de Janeiro, Brazil; 
CHIP-URG Helminthological Collection of the Laboratory of Ichthyoparasitology, University of Rio Grande, Rio Grande, Rio Grande do Sul, Brazil;

CHMLP/MLP Helminthological Collection of the Museum of La Plata, La Plata, Buenos Aires, Argentina;

CMNPA Canadian Museum of Nature Invertebrate Collections-Parasites, Ottawa, Canada;

CNHE National Collection of Helminths, Institute of Biology, National Autonomous University of Mexico, Mexico City, Mexico;

CZACC Helminthological Collection of the Zoological Collections, Institute of Ecology and Systematic, Havana, Cuba;

FCAV/UNESP Helminthological Collection of the Parasitic Diseases Laboratory, Department of Preventive Veterinary Medicine, Paulista State University, São Paulo, Brazil;

HWML Harold W. Manter Laboratory, University of Nebraska State Museum, Lincoln, Nebraska, USA;

INPA National Institute for Amazon Research, Manaus, Amazonas, Brazil;

IPCAS/IPCR/ASCR Institute of Parasitology, Academy of Sciences of the Czech Republic, České Budějovice, Czech Republic;

MNHN/INVE National Museum of Natural History, Paris, France;

MNRJ Carcinological Collection of the National Museum of Rio de Janeiro, Rio de Janeiro, Brazil;

RIT Royal Belgian Institute of Natural Sciences, Brussels, Belgium;

RMCA Collection of the Royal Museum of Central Africa, Tervuren, Belgium;

USNM United States National Museum, Smithsonian Institution, Washington, D.C, USA;

USNPC United States National Parasitological Collection, Beltsville, Maryland, USA.

\section{Results and discussion}

This database and bibliographic survey provides the diversity types of Acanthocephala, Nematoda and other non-helminth phyla, such as Cnidaria (Myxozoa), Annelida (Polychaeta and Hirudinea) and Arthropoda (Copepoda), in CHIOC, from Brazil and other countries of the world, for a period of more than 35 years of parasitological studies. This catalogue adds 38 primary types of Acanthocephala, represented by eight species distributed among eight families and eight genera; approximately 420 primary types of Nematoda, represented by 99 species, distributed across 36 families and 64 genera; four primary types of Myxozoa, represented by one family, two genera and two species; eight primary types of one species of Polychaeta and six primary types of one species of Hirudinea; and 22 primary types of Copepoda, represented 
by five species, distributed in three families, and four genera. The most representative families were the nematode families Cucullanidae, with 18 species, followed by Pharyngodonidae, with seven.

One hundred and sixteen parasites of 101 species of vertebrate and eight species of invertebrate hosts were catalogued. The invertebrate hosts were beetles, diplopods, and crustaceans. Most of the type species recorded (47\%) were parasites of bony fishes, such as those of Monogenoidea, Trematoda, and Cestoda (Lopes et al. 2016, 2017). Only one species was a parasite of cartilaginous fishes. The hosts of parasite species of Tetrapoda were frogs, birds, reptiles, and mammals. Cricetidae (Rodentia) was the host family that exhibited the greatest diversity of parasites, with ten species.

Among the Acanthocephala listed, five species were parasites of bony fishes, one species was a parasite of frogs (Bufonidae), one species was a parasite of birds (Phalacrocoracidae), and one species was a parasite of crab-eating foxes (Canidae). Among the nematodes, five species were parasites of beetles (Passalidae) and two were parasites of diplopods (Rhinocricidae and Spirobolellidae). Species of nematode parasites of fishes included 41 of bony fishes and only one of cartilaginous fishes (Arhynchobatidae). Three nematode species were parasites of frogs (Bufonidae). The nematodes parasites of reptiles included five species parasitic on the suborder Autarchoglossa (Gymnophthalmidae, Scincidae and Teiidae), five parasites of snakes (Boidae, Colubridae, and Viperidae), and three parasites of lizards (Hoplocercidae, Liolaemidae, and Tropiduridae). Nine nematode species were parasites of birds (Ardeidae, Bucconidae, Cuculidae, Dendrocolaptidae, Opisthocomidae, Threskiornithidae, Tinamidae, and Tyrannidae). Species of nematode parasites of mammals included 16 parasites of rodents (Caviidae, Cricetidae, Dasyproctidae, Echimyidae, and Muridae), four parasites of the order Carnivora (Canidae, Felidae, and Mustelidae), two parasites of armadillos (Dasypodidae), two parasites of opossums (Didelphidae), and one parasite of bats (Molossidae). Myxozoans and copepods parasitized bony fishes. The single polychaeta species was a parasite of freshwater crayfish (Parastacidae) and the single Hirudinea species was a parasite of turtles (Podocnemididae).

Most of the species catalogued were collected in Brazil (88\%), coming from all regions of the country. Other countries with cataloged material are from America (United States, Mexico, Panama, Colombia, Venezuela, Bolivia, and Argentina), the Caribbean (Cuba) and Africa (Democratic Republic of Congo). This third part of the catalogue of type material housed in CHIOC from 1979 to 2016 includes, in total, 423 species of parasites from 251 different hosts caught in almost all Brazilian states, and almost all continents, with the exception of the Middle East and Oceania (Lopes et al. 2016, 2017). Most of the deposits were made by Brazilian researchers, but foreigners also made a significant contribution, expanding and diversifying the country's known parasitological heritage. All these data testify to the importance of $\mathrm{CHIOC}$ and its recognition on the world stage as a depositary, and serve as testimony of helminth parasite biodiversity, thereby placing CHIOC among the most significant collections of the world. 
List of type species

Phylum Acanthocephala Kohlreuther, 1771

Class Archiacanthocephala Meyer, 1931

Order Oligacanthorhynchida Petrochenko, 1956

Oligacanthorhynchidae Southwell \& Macfie, 1925

Prosthenorchis Travassos, 1915

Prosthenorchis cerdocyonis Gomes, Olifiers, Souza, Barbosa, D'Andrea, \& Maldonado Jr., 2015

Type host. Cerdocyon thous (Linnaeus, 1766) (Carnivora: Canidae).

Infection site. Small intestine.

Type locality. Brazil, Mato Grosso do Sul State, Nhumirim Ranch (1859'S, 56³9'W).

Holotype. ô CHIOC 35804 a.

Paratypes. CHIOC 35804 b (allotype + ), 30812 c $(5 q q, 6 ふ ึ)$.

Reference. Gomes et al. (2015).

Class Eoacanthocephala Van Cleave, 1936

Order Gyracanthocephala Van Cleave, 1936

Quadrigyridae Van Cleave, 1920

Machadosentis Noronha, 1992

*Machadosentis travassosi Noronha, 1992

Type host. Gymnothorax ocellatus Agassiz, 1831 (Osteichthyes: Muraenidae).

Infection site. Intestine.

Type locality. Brazil, Bahia State, Arembepe.

Syntypes. CHIOC $32742 \mathrm{a}-\mathrm{b}(\hat{\jmath})$, c (ㅇ).

Remarks. CHIOC $32742 \mathrm{a}-\mathrm{c}$ referred as type material in the original description.

Reference. Noronha (1992).

Order Neoechinorhynchida Southwell \& MacFie, 1925

Neoechinorhynchidae (Ward, 1917) Van Cleave, 1928

Neochinorhynchus Stiles \& Hassal, 1905

Neoechinorhynchus pimelodi Brasil-Sato \& Pavanelli, 1998

Type host. Pimelodus maculatus Lacépède, 1803 (Osteichthyes: Pimelodidae).

Infection site. Anterior intestine.

Type locality. Brazil, Minas Gerais State, São Francisco River basin, Três Marias.

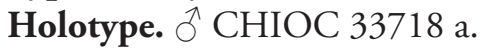


Paratypes. CHIOC 33718 b (allotype $q$ ), c, e (ð̋̂), d ()).

Remarks. Other paratypes deposited in USNPC.

Reference. Brasil-Sato and Pavanelli (1998).

Class Palaeacanthocephala Meyer, 1931

Order Echinorhynchida Southwell \& MacFie, 1925

Arhythmacanthidae Yamaguti, 1935

Heterosentis Van Cleave, 1931

Heterosentis brasiliensis Vieira, Felizardo \& Luque, 2009

Type host. Pseudopercis numida Miranda-Ribeiro, 1903 (Osteichthyes: Pinguipedidae).

Infection site. Intestine.

Typelocality. Brazil, Rio de Janeiro State, Cabo Frio (2252'43.26"S, 42¹'11.55"W).

Holotype. ô CHIOC 37103.

Paratypes. CHIOC 36672 (ð̊), 37104 (allotype + ), 37105 ().

Reference. Vieira et al. (2009).

Diplosentidae Tubangui \& Masilungan, 1937

Amapacanthus Salgado-Maldonado \& Santos, 2000

*Amapacanthus amazonicus Salgado-Maldonado \& Santos, 2000

Type host. Sciades passany (Valenciennes, 1840) [= Arius passany] (Osteichthyes: Ariidae).

Infection site. Intestine.

Type locality. Brazil, Amapá State, Maraca Island $\left(2^{\circ} 0^{\prime} \mathrm{N}, 50^{\circ} 24^{\prime} \mathrm{W}\right)$.

Holotype. ¿̊ CHIOC 34199 a.

Paratypes. CHIOC 34199 b (allotype $q$ ), c-j ( $q$ q).

Remarks. Other paratypes deposited in CNHE and BMNH.

Reference. Salgado-Maldonado and Santos (2000).

Echinorhynchidae Cobbold, 1876

Acanthocephalus Koelreuter, 1771

Acanthocephalus ula Lent \& Santos, 1989

Type host. Atelopus oxyrhynchus Boulenger, 1903 (Amphibia: Bufonidae).

Infection site. Small intestine.

Type locality. Venezuela, Mérida.

Holotype. $\widehat{\sigma}$ CHIOC 32173.

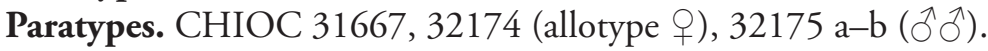

Reference. Lent and Santos (1989). 
Gymnorhadinorhynchidae Braicovich, Lanfranchi, Farber, Marvaldi, Luque \& Timi, 2014

Gymnorhadinorhynchus Braicovich, Lanfranchi, Farber, Marvaldi, Luque \& Timi, 2014

*Gymnorhadinorhynchus decapteri Braicovich, Lanfranchi, Farber, Marvaldi, Luque \& Timi, 2014

Type host. Decapterus punctatus (Cuvier, 1829) (Osteichthyes: Carangidae).

Infection site. Intestine.

Type locality. Brazil, Rio de Janeiro State, Cabo Frio $\left(22^{\circ} 53^{\prime} \mathrm{S}, 42^{\circ} 00^{\prime} \mathrm{W}\right)$.

Holotype. ${ }^{A}$ CHIOC 35941 a.

Paratypes. CHIOC 35941 b (allotype +), 35941 c (ð)), d-e (우).

Remarks. Two additional paratypes deposited in the IPCAS collection.

Reference. Braicovich et al. (2014).

Order Polymorphida Petrochenko, 1956

Polymorphidae Meyer, 1931

Andracantha Schmidt, 1975

Andracantha tandemtesticulata Monteiro, Amato \& Amato, 2006

Type host. Phalacrocorax brasilianus (Gmelin, 1789) (Aves: Phalacrocoracidae).

Infection site. Small and large intestine.

Type locality. Brazil, Rio Grande do Sul State, Guaíba Lake, Guaíba $\left(30^{\circ} 00^{\prime}\right.$ S, $\left.51^{\circ} 15^{\prime} \mathrm{W}\right)$.

Holotype. $\delta^{\lambda}$ CHIOC 36630 a.

Paratypes. CHIOC 36630 b (allotype + ), 36630 c (q).

Reference. Monteiro et al. (2006).

Phylum Nematoda Potts, 1932

Class Enoplea Inglis, 1983

Order Trichinellida Hall, 1916

Capillariidae Railliet, 1915

Capillostrongyloides Freitas \& Lent, 1935

Capillostrongyloides arapaimae Santos, Moravec \& Venturieri, 2008

Type host. Arapaima gigas (Schinz, 1822) (Osteichthyes: Arapaimidae).

Infection site. Anterior part of intestine and pyloric caeca.

Type locality. Brazil, Pará State, Mexiana Island (Amazon River delta), natural canals and breeding tanks of fish farm at Santo Ambrósio Farm (00 0 0 '30"S, 49³4'50"W). 
Holotype. $\lesssim$ CHIOC 35559 a.

Paratypes. CHIOC 35559 b (allotype +), 35559 c-d ( ${ }^{\lambda}$, ㅇ).

Reference. Santos et al. (2008a).

Pseudocapillaria (Ichthyocapillaria) Moravec, 1982

Pseudocapillaria (Ichthyocapillaria) maricaensis Rodrigues, 1992

Type host. Liolaemus lutzae Mertens, 1938 (Iguania: Liolaemidae).

Infection site. Small intestine.

Type locality. Brazil, Rio de Janeiro State, Maricá.

Holotype. ${ }^{\lambda}$ CHIOC 32796 a.

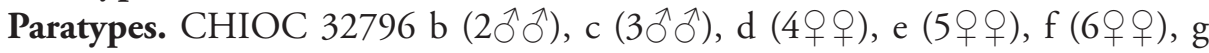
(7우우), h (8웅), i (9우우).

Reference. Rodrigues (1992).

Trichuridae Ransom, 1911

Trichuris Roederer, 1761

Trichuris thrichomysi Lopes Torres, Nascimento, Menezes, Garcia, Santos, Maldonado Jr., Miranda, Lanfredi \& Souza, 2011

Type host. Thrichomys apereoides Lund, 1839 (Rodentia: Echimyidae).

Infection site. Cecum.

Type locality. Brazil, Minas Gerais State, Capitão Andrade $\left(19^{\circ} 02^{\prime} 14 " S\right.$, $\left.41^{\circ} 49^{\prime} 59^{\prime \prime W}\right)$.

Holotype. $\widehat{o}$ CHIOC 35210 a.

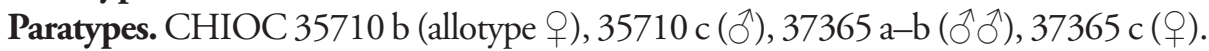

Reference. Lopes Torres et al. (2011).

Trichuris travassosi Gomes, Lanfredi, Pinto \& Souza, 1992

Type host. Oligoryzomys nigripes (Olfers, 1818) [= Oryzomys nigripes] (Rodentia: Cricetidae).

Infection site. Large intestine.

Type locality. Brazil, Rio Grande do Sul State, Arvorezinha ( $\left.28^{\circ} 45^{\prime} \mathrm{S}, 52^{\circ} 15^{\prime} \mathrm{W}\right)$.

Holotype. $\widehat{O}$ CHIOC 32790 a.

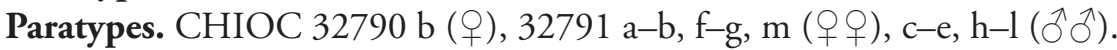

Reference. Gomes et al. (1992). 
Class Chromadorea Inglis, 1983

Order Rhabditida Chitwood, 1933

Acuariidae Railliet, Henry \& Sissoff, 1912

Deliria Vicente, Pinto \& Noronha, 1980

*Deliria gomesae Vicente, Pinto \& Noronha, 1980

Type host. Pitangus sulphuratus (Linnaeus, 1766) (Aves: Tyrannidae).

Infection site. Stomach.

Type locality. Brazil, Rio de Janeiro State, Rio de Janeiro, Raimundo Island.

Holotype. O CHIOC 31780 a.

Paratypes. CHIOC 31780 b-c, g-h (우), d-f (

Remarks. There is no paratype " $i$ " as indicated in the original description, which was a mistake.

Reference. Vicente et al. (1980).

Anisakidae Railliet \& Henry, 1912

Goezia Zeder, 1800

Goezia brasiliensis Moravec, Kohn \& Fernandes, 1994

Type host. Brycon hilarii (Valenciennes, 1850) (Osteichthyes: Bryconidae).

Infection site. Stomach.

Type locality. Brazil, Paraná State, Paraná River, Foz do Iguaçú.

Paratype. + CHIOC 32961.

Remarks. Holotype and allotype deposited in the IPCAS collection.

Reference. Moravec et al. (1994).

Goezia leporini Martins \& Yoshitoshi, 2003

Type host. Leporinus macrocephalus Garavello \& Britski, 1988 (Osteichthyes: Anostomidae).

Infection site. Stomach.

Type locality. Brazil, São Paulo State, Batatais.

Holotype. $\widehat{\alpha}$ CHIOC 34675 a.

Paratypes. CHIOC 34675 b (allotype +), 34675 c (ふぶ), 34675 d (우).

Reference. Martins and Yoshitoshi (2003). 
Pseudanisakis Layman \& Borovkova, 1926

Pseudanisakis sulamericana Santos, Lent \& Gibson, 2004

Type host. Rioraja agassizii (Müller \& Henle, 1841) (Chondrichthyes: Arhynchobatidae). Infection site. Intestine.

Type locality. Brazil, São Paulo State, off Ubatuba $\left(23^{\circ} 30^{\prime} \mathrm{S}, 45^{\circ} 00^{\prime} \mathrm{W}\right)$.

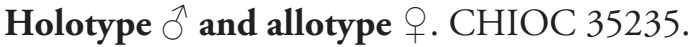

Paratypes. CHIOC 35236, 35237.

Remarks. CHIOC 35237 collected from Psammobatis extenta (Garman, 1913) (Arhynchobatidae). Other paratypes deposited in the BMHN collection.

Reference. Santos et al. (2004).

Raphidascaris (Sprentascaris) (Petter \& Cassone, 1984) Moravec, Kohn \& Fernandes, 1990

Raphidascaris (Sprentascaris) lanfrediae Melo, Santos, Giese, Santos \& Santos, 2011

Type host. Satanoperca jurupari (Heckel, 1840) (Osteichthyes: Cichlidae).

Infection site. Intestine.

Type locality. Brazil, Pará State, Belém, Guamá River (012ㄱ'21"S, 48³0'14"W).

Holotype $\overparen{T}$. CHIOC 35714 a.

Paratypes. CHIOC $35714 \mathrm{~b}-\mathrm{c}\left(5+9\right.$, $\left.4 \mathrm{O}^{\Uparrow} \mathrm{o}^{\lambda}\right)$.

Remarks. Paratype from CHIOC cited as "35716" in the original description due to a mistake.

Reference. Melo et al. (2011).

Raphidascaroides Railliet \& Henry, 1915

Raphidascaroides moraveci Pereira, Tavares, Scholz \& Luque, 2015

Type host. Platydoras armatulus (Valenciennes, 1840) (Osteichthyes: Doradidae).

Infection site. Intestine.

Type locality. Brazil, Mato Grosso do Sul State, Miranda River (19 $\left.34^{\prime} \mathrm{S}, 57^{\circ} 00^{\prime} \mathrm{W}\right)$.

Holotype $\delta$ and allotype $q$. CHIOC 36728 a.

Paratypes. CHIOC 36728 b ( $3 \hat{\jmath} \hat{\jmath}, 3$ 우 $ᄋ$ ), c (hologenophore).

Remarks. Other paratypes deposited in the IPCAS collection.

Reference. Pereira et al. (2015). 
Aproctidae Yorke \& Maplestone, 1926

Aprocta Linstow, 1883

Aprocta brevipenis Rodrigues \& Rodrigues, 1980

Type host. Guira guira (Gmelin, 1788) (Aves: Cuculidae).

Infection site. Ocular cavity.

Type locality. Brazil, São Paulo State, Ilha Seca.

Holotype. $\widehat{O}$ CHIOC 31808 a.

Paratypes. CHIOC 31808 b (allotype $q$ ), $31808 \mathrm{c}-\mathrm{d}$ (웅) .

Reference. Rodrigues and Rodrigues (1980).

Ascaridiidae Travassos, 1919

Ophidascaris Baylis, 1920

Ophidascaris durissus Panizzutti, Santos, Vicente, Muniz-Pereira \& Pinto, 2003

Type host. Crotalus durissus Linnaeus, 1758 (Serpentes: Viperidae).

Infection site. Stomach.

Type locality. Brazil, Paraná State, Itaipú Binacional Reserve, Foz do Iguaçú $\left(24^{\circ} 05^{\prime}-25^{\circ} 33^{\prime} \mathrm{S}, 54^{\circ} 00^{\prime}-54^{\circ} 37^{\prime} \mathrm{W}\right)$.

Holotype. CHIOC 34937 a.

Paratypes. CHIOC 34937 b-f.

Reference. Panizzuti et al. (2003).

Ophidascaris tuberculatum Siqueira, Panizzutti, Muniz-Pereira \& Pinto, 2005

Type host. Bothrops jararaca (Wied-Neuwied, 1824) (Serpentes: Viperidae).

Infection site. Stomach.

Type locality. Brazil, Rio de Janeiro State, Petrópolis, Serra das Araras (22³0'39"S, $\left.43^{\circ} 11^{\prime} 4 " \mathrm{~W}\right), 857 \mathrm{~m}$ high.

Holotype. $\lesssim$ CHIOC 36232 a.

Paratypes. CHIOC 35315, 36232 b (allotype ) ), 36232 c (ㅇ), d (anterior extremity).

Reference. Siqueira et al. (2005).

Aspidoderidae Skrjabin \& Schikhobalova, 1947

Aspidodera Railliet \& Henry, 1912

Aspidodera sogandaresi Jiménez-Ruiz, Gardner \& Varela-Stokes, 2006

Type host. Dasypus novemcinctus Linnaeus, 1758 (Cingulata: Dasypodidae). 
Infection site. Large intestine.

Type locality. United States, Texas, El Pedregal, 18 miles north by road (U.S. 281) from Lampasas (31 $\left.19^{\prime} 34^{\prime \prime N}, 98^{\circ} 09^{\prime} 33^{\prime \prime W}\right), 311 \mathrm{~m}$ high.

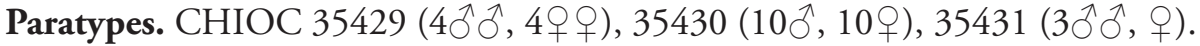

Remarks. There are two paratypes in CHIOC collected from Mexico, but there are some inconsistencies with regard to the collecting localities in the original description and the cataloging data. Holotype, allotype, and other paratypes are deposited in the HWML collection. Additional paratypes are deposited in CMNPA, CNHE, and USNM.

Reference. Jiménez-Ruiz et al. (2006).

\section{Aspidodera vicentei Pinto, Kohn, Fernandes \& Mello, 1982}

Type host. Nectomys squamipes (Brants, 1827) (Rodentia: Cricetidae).

Infection site. Small intestine.

Type locality. Brazil, Goiás State, Formosa.

Holotype. $\widehat{o}$ CHIOC 31879 a.

Paratypes. CHIOC 31879 b-f.

Reference. Pinto et al. (1982).

\section{Camallanidae Railliet \& Henry, 1915}

Camallanus Railliet \& Henry, 1915

\section{Camallanus acaudatus Ferraz \& Thatcher, 1990}

Type host. Osteoglossum bicirrhosum (Cuvier, 1829) (Osteichthyes: Osteoglossidae).

Infection site. Intestine.

Type locality. Brazil, Amazonas State, Anavilhanas Archipelago, Negro River.

Paratypes. CHIOC $32557 \mathrm{a}-\mathrm{b}(\hat{o}$,, ).

Remarks. Holotype and allotype deposited in the INPA collection. CHIOC numbers were not included in the original description.

Reference. Ferraz and Thatcher (1990).

Camallanus maculatus Martins, Garcia, Piazza \& Ghiraldelli, 2007

Type host. Xiphophorus maculatus (Günther, 1866) (Osteichthyes: Poeciliidae).

Infection site. Intestine.

Type locality. Brazil, São Paulo State, Araraquara.

Holotype $\hat{\sigma}$, allotype $q$ and paratypes. CHIOC 35283.

Reference. Martins et al. (2007). 
Paracamallanus Yorke \& Maplestone, 1926

Paracamallanus amazonensis Ferraz \& Thatcher, 1992

Type host. Hypophthalmus edentatus Spix \& Agassiz, 1829 (Osteichthyes: Pimelodidae). Infection site. Intestine.

Type locality. Brazil, Amazonas State, Marchantaria Island, Solimóes River.

Paratypes. CHIOC 32756 (つึ), 32757 (†).

Remarks. Holotype, allotype and other paratypes deposited in the collection of INPA. CHIOC numbers were not included in the original description.

Reference. Ferraz and Thatcher (1992).

Procamallanus (Procamallanus) (Baylis, 1923) Ali, 1957

Procamallanus (Procamallanus) annipetterae Kohn \& Fernandes, 1988

Type host. Hypostomus albopunctatus (Regan, 1908) (Osteichthyes: Loricariidae).

Infection site. Intestine.

Type locality. Brazil, Paraná State, Iguaçú River, Hydroelectric Power station Salto Osório.

Holotype. $₫$ CHIOC 32430 a.

Paratype. CHIOC $32430 \mathrm{~b}$ (allotype $q$ ).

References. Kohn and Fernandes (1988a, b).

Procamallanus (Spirocamallanus) (Olsen, 1952) Moravec \& Sey, 1988

Procamallanus (Spirocamallanus) belenensis Giese, Santos \& Lanfredi, 2009

Type host. Ageneiosus ucayalensis Castelnau, 1855 (Osteichthyes: Auchenipteridae).

Infection site. Intestine.

Type locality. Brazil, ParáState, Belém, Guajará Bay $\left(1^{\circ} 15^{\prime}-1^{\circ} 29^{\prime} \mathrm{S}, 48^{\circ} 32^{\prime}-48^{\circ} 29^{\prime} \mathrm{W}\right)$.

Holotype. $\widehat{o}$ CHIOC 35604 a.

Paratypes. CHIOC 35604 b (allotype + ), 35604 c.

Reference. Giese et al. (2009).

Procamallanus (Spirocamallanus) pintoi Kohn \& Fernandes, 1988

Type host. Corydoras paleatus (Jenyns, 1842) (Osteichthyes: Callichthyidae).

Infection site. Intestine.

Type locality. Brazil, Paraná State, Iguaçú River, Hydroelectric Power station Salto Osório. 
Holotype. đิ CHIOC 32432 a.

Paratypes. CHIOC 32431 a (allotype ㅇ) , 32431 b (đ̊), 32432 b-c (우).

Reference. Kohn and Fernandes (1988a).

\section{Carnoyidae Filipjev, 1934}

Carnoya Gilson, 1898

Carnoya isabelica García \& Morffe, 2014

Type host. Nesobolus piedra Pérez-Asso, 1996 (Diplopoda: Rhinocricidae).

Infection site. Hind gut.

Type locality. Cuba, Santiago de Cuba province, La Gran Piedra, La Isabelica, $\left(20^{\circ} 00^{\prime} 32.68^{\prime \prime} \mathrm{N}, 75^{\circ} 37^{\prime} 18.8^{\prime \prime} \mathrm{W}\right)$.

Paratypes. CHIOC $38210 \mathrm{a}-\mathrm{b}(2 \hat{\jmath} \widehat{\jmath}, 3$ 웅) $)$.

Remarks. Holotype female and other paratypes males and females deposited in CZACC.

Reference. García and Morffe (2014).

Cosmocercidae Railliet, 1916

Cosmocercoides Wilkie, 1930

Cosmocercoides sauria Ávila, Strüssmann \& Silva, 2010

Type host. Iphisa elegans Gray, 1851 (Autarchoglossa: Gymnophthalmidae).

Infection site. Large intestine.

Type locality. Brazil, Mato Grosso State, São Domingos Valley $\left(15^{\circ} 07^{\prime} \mathrm{S}, 58^{\circ} 58^{\prime} \mathrm{W}\right)$.

Holotype. ¿ै CHIOC 35654 a.

Paratype. CHIOC 35654 b (allotype 9 ).

Remarks. Three paratypes deposited in CHIBB.

Reference. Ávila et al. (2010).

Crenosomatidae Skrjabin, 1933

Crenosoma Molin, 1861

Crenosoma brasiliense Vieira, Muniz-Pereira, Lima, Moraes Neto, Guimarães \& Luque, 2012

Type host. Galictis cuja (Molina, 1782) (Carnivora: Mustelidae).

Infection site. Bronchi and bronchioles.

Type locality. Brazil, Minas Gerais State, Juiz de Fora $\left(21^{\circ} 76^{\prime} \mathrm{S}, 43^{\circ} 21^{\prime} \mathrm{W}\right)$.

Holotype. ¿ै CHIOC 35813 a. 
Paratypes. CHIOC 35813 b (allotype ㅇ), 35813 c (ふึ), 35813 d (2웅).

Remarks. One paratype male and two females deposited in the IPCAS collection.

Reference. Vieira et al. (2012).

\section{Cucullanidae Cobbold, 1864}

Cucullanus Mueller, 1777

Cucullanus ageneiosi Giese, Lanfredi \& Santos, 2010

Type host. Ageneiosus ucayalensis

Infection site. Small intestine.

Type locality. Brazil, Pará State, Belém, Guajará Bay $\left(1^{\circ} 15^{\prime}-1^{\circ} 29^{\prime} \mathrm{S}, 48^{\circ} 32-48^{\circ} 29^{\prime} \mathrm{W}\right)$.

Holotype. $\widehat{o}$ CHIOC 35657 a.

Paratype. CHIOC 35657 b (allotype ${ }^{\text {) }}$.

Reference. Giese et al. (2010).

Cucullanus brevicaudatus Pereira, Vieira \& Luque, 2014

Type host. Balistes capriscus Gmelin, 1789 (Osteichthyes: Balistidae).

Infection site. Intestine.

Type locality. Brazil, Rio de Janeiro State, Angra dos Reis Bay $\left(23^{\circ} 00^{\prime} \mathrm{S}, 44^{\circ}-10^{\prime} \mathrm{W}\right)$.

Holotype. ${ }^{A}$ CHIOC 35896 a.

Paratypes. CHIOC 35896 b (allotype 우), 35897 ( $3 \hat{\jmath}$, 3 웅) .

Remarks. Two paratypes males and two females deposited in the IPCAS collection.

Reference. Pereira et al. (2014c).

Cucullanus brevispiculus Moravec, Kohn \& Fernandes, 1993

Type host. Auchenipterus nuchalis (Spix \& Agassiz, 1829) (Osteichthyes: Auchenipteridae).

Infection site. Intestine.

Type locality. Brazil, Paraná State, Foz do Iguaçú, Reservoir of the hydroelectric power station of Itaipú.

Holotype. đ̊ CHIOC 32951.

Remarks. One specimen deposited, no paratypes.

Reference. Moravec et al. (1993).

Cucullunus cassinensis Pereira Jr. \& Costa, 1996

Type host. Micropogonias furnieri (Desmarest, 1823) (Osteichthyes: Sciaenidae).

Infection site. Digestive tract. 
Type locality. Brazil, Rio Grande do Sul State, Rio Grande.

Holotype. + CHIOC 33655.

Paratype. CHIOC 33656 (allotype đ).

Remarks. Other paratypes deposited in CHIP-URG.

Reference. Pereira Jr. and Costa (1996).

Cucullanus gastrophysi Pereira, Vieira \& Luque, 2015 in Vieira et al. (2015)

Type host. Lophius gastrophysus Miranda-Ribeiro, 1915 (Osteichthyes: Lophiidae).

Infection site. Intestine.

Type locality. Brazil, Rio de Janeiro State, Cabo Frio $\left(22^{\circ} 52^{\prime} \mathrm{S}, 42^{\circ} 01^{\prime} \mathrm{W}\right)$.

Holotype. ${ }^{\lambda}$ CHIOC 36745 a.

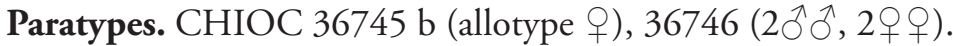

Remarks. One paratype male and one female deposited in the IPCAS collection.

Reference. Vieira et al. (2015).

Cucullanus protudens Pereira, Vieira \& Luque, 2015 in Vieira et al. (2015)

Type host. Pagrus pagrus (Linnaeus, 1758) (Osteichthyes: Sparidae).

Infection site. Intestine.

Type locality. Brazil, Rio de Janeiro State, Cabo Frio $\left(22^{\circ} 52^{\prime} \mathrm{S}, 42^{\circ} 01^{\prime} \mathrm{W}\right)$.

Holotype. ${ }^{A}$ CHIOC 36747 a.

Paratypes. CHIOC 36747 b (allotype + ), 36748 (ㅇ).

Reference. Vieira et al. (2015).

Cucullanus pseudopercis Pereira, Vieira \& Luque, 2015 in Vieira et al. (2015)

Type host. Pseudopercis semifasciata (Cuvier, 1829)

Infection site. Intestine.

Type locality. Brazil, Rio de Janeiro State, Cabo Frio $\left(22^{\circ} 52^{\prime} \mathrm{S}, 42^{\circ} 01^{\prime} \mathrm{W}\right)$.

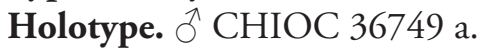

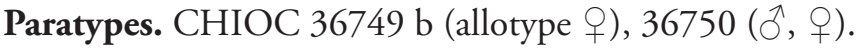

Remarks. One paratype male deposited in the IPCAS collection.

Reference. Vieira et al. (2015).

Cucullanus grandistomis (Ferraz \& Thatcher, 1988)

Type host. Oxydoras niger (Valenciennes, 1821) (Osteichthyes: Doradidae).

Infection site. Intestine.

Type locality. Brazil, Amazonas State, Solimóes River near Manaus. 
Paratypes. CHIOC $32456 \mathrm{a}-\mathrm{b}(\widehat{\jmath}$,, ) .

Remarks. Holotype and allotype deposited in the INPA collection. CHIOC numbers were not included in the original description.

Reference. Ferraz and Thatcher (1988).

\section{Cucullanus heliomartinsi Moreira, Rocha \& Costa, 2000}

Type host. Trachelyopterus striatulus (Steindachner, 1877) [= Parauchenipterus striatulus] (Osteichthyes: Auchenipteridae).

Infection site. Intestine.

Type locality. Brazil, Minas Gerais State, Central Lake of the State Park of Rio Doce $\left(19^{\circ} 50^{\prime} \mathrm{S}, 42^{\circ} 35^{\prime} \mathrm{W}\right)$.

Holotype. $ð$ CHIOC 33863 a.

Paratype. CHIOC 33863 b (allotype 9 ).

Reference. Moreira et al. (2000).

Cucullanus pimelodellae Moravec, Kohn \& Fernandes, 1993

Type host. Pimelodella lateristriga (Lichtenstein, 1823) (Osteichthyes: Heptapteridae). Infection site. Intestine.

Type locality. Brazil, Paraná State, Guaíra.

Paratype. CHIOC 32826 ( ঐ).

Remarks. Holotype deposited in the ASCR collection.

Reference. Moravec et al. (1993).

Cucullanus pinnai pterodorasi Moravec, Kohn \& Fernandes, 1997

Type host. Pterodoras granulosus (Valenciennes, 1821) (Osteichthyes: Doradidae).

Infection site. Intestine.

Type locality. Brazil, Paraná State, Guaíra (Paraná River basin), Reservoir of Itaipú.

Holotype ${ }^{\lambda}$, allotype $q$ and paratype. CHIOC 33532.

Remarks. Paratype from CHIOC cited as " 33697 " in the original description due to a mistake. Other paratypes deposited in the ASCR collection.

Reference. Moravec et al. (1997).

Cucullanus pseudoplatystomae Moravec, Kohn \& Fernandes, 1993

Type host. Pseudoplatystoma corruscans (Spix \& Agassiz, 1829) (Osteichthyes: Pimelodidae).

Infection site. Intestine. 
Type locality. Brazil, Paraná State, Paraná River, Guaíra.

Holotype. $\widehat{O}$ CHIOC 32829 a.

Paratypes. CHIOC 32829 b (allotype +), 32829 c-d, 32910, 32913, 32914.

Remarks. Other paratypes deposited in the ASCR collection.

Reference. Moravec et al. (1993).

\section{Cucullanus rhamphichthydis Moravec, Kohn \& Fernandes, 1997}

Type host. Rhamphichthys rostratus (Linnaeus, 1766) (Osteichthyes: Rhamphichthyidae).

Infection site. Intestine.

Type locality. Brazil, Paraná State, Santa Helena, reservoir of the hydroelectric power station of Itaipú.

Holotype. + CHIOC 33533.

Paratypes. CHIOC 33533 (2웅)

Remarks. Type material from CHIOC cited as " 33716 " in the original description due to a mistake. Other paratypes deposited in the ASCR collection.

Reference. Moravec et al. (1997).

Cucullanus tucunarensis Lacerda, Takemoto, Marchiori, Martins \& Pavanelli, 2013

Type host. Cichla piquiti Kullander \& Ferreira, 2006 (Osteichthyes: Cichlidae).

Infection site. Intestine.

Type locality. Brazil, Tocantins State, Lajeado reservoir (1066'55"S, 4842'36"W).

Holotype. $q$ CHIOC 35877 a.

Paratype. CHIOC 35877 b (allotype ${ }^{\Uparrow}$ ).

Remarks. Other paratypes deposited in CNHE.

Reference. Lacerda et al. (2013).

Dichelyne (Cucullanellus) (Tornquist, 1931)

Dichelyne (Cucullanellus) sciaenidicola Timi, Lanfranchi, Tavares \& Luque, 2009

Type host. Umbrina canosai Berg, 1895 (Osteichthyes: Sciaenidae).

Infection site. Posterior end of intestine.

Type locality. Argentina, Buenos Aires Province, Mar del Plata $\left(38^{\circ} 08^{\prime} \mathrm{S}, 57^{\circ} 32^{\prime} \mathrm{W}\right)$.

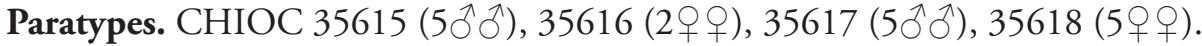

Remarks. CHIOC 35617 and 35618 collected in Pedra de Guaratiba $\left(23^{\circ} 01^{\prime} S\right.$, $\left.43^{\circ} 38^{\prime} \mathrm{W}\right)$, Rio de Janeiro (Rio de Janeiro State, Brazil) from the sciaenid fish Micropogonias furnieri. Holotype, allotype, and other paratypes deposited in CHMLP.

Reference. Timi et al. (2009). 
Dichelyne (Cucullanellus) tornquisti Paschoal, Vieira, Cezar \& Luque, 2014

Type host. Orthopristis ruber (Cuvier, 1830) (Osteichthyes: Haemulidae).

Infection site. Intestine.

Type locality. Brazil, coast of the Rio de Janeiro State $\left(21-23^{\circ} \mathrm{S}, 42-45^{\circ} \mathrm{W}\right)$.

Holotype. 0 CHIOC 35894 a.

Paratypes. CHIOC 35894 b (allotype ㅇ), 35895 (2ภㅊ, 2우).

Remarks. One paratype male and two females deposited in the IPCAS collection.

Reference. Paschoal et al. (2014).

Dichelyne (Dichelyne) Jägerskiöld, 1902

Dichelyne (Dichelyne) pimelodi Moravec, Kohn \& Fernandes, 1997

Type host. Pimelodus maculatus

Infection site. Intestine.

Type locality. Brazil, Paraná State, Guaíra (Paraná River basin), Reservoir of Itaipú.

Holotype. $\widehat{\jmath}$ CHIOC 33534.

Remarks. Holotype cited as " 33717 " in the original description due to a mistake.

Reference. Moravec et al. (1997).

Dichelyne (Dichelyne) micropogonii Pereira Jr. \& Costa, 1996

Type host. Micropogonias furnieri

Infection site. Digestive tract.

Type locality. Brazil, Rio Grande do Sul State, Rio Grande.

Holotype. ㅇ CHIOC 33649.

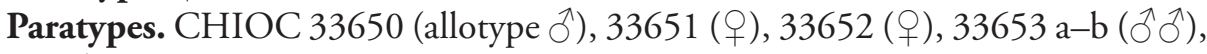
$33654\left({ }^{\top}\right)$.

Remarks. Other paratypes deposited in CHIP-URG.

Reference. Pereira Jr. and Costa (1996).

Cystidicolidae Skrjabin, 1946

Ascarophis van Beneden, 1871

Ascarophis brasiliensis Pinto, Vicente \& Noronha, 1984

Type host. Trachinotus carolinus (Linnaeus, 1766) (Osteichthyes: Carangidae).

Infection site. Stomach. 
Type locality. Brazil, Rio de Janeiro State, Araruama (22 $\left.52^{\prime} 23^{\prime \prime S}, 42^{\circ} 20^{\prime} 20^{\prime \prime W}\right)$. Holotype. $\widehat{o}$ CHIOC 32032 a.

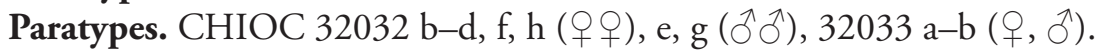

Reference. Pinto et al. (1984).

Comephoronema Layman, 1933

Comephoronema multipapillatum Pereira, Pereira \& Luque, 2014

Type host. Holocentrus adscensionis (Osbeck, 1765) (Osteichthyes: Holocentridae).

Infection site. Anterior intestine and caecum.

Type locality. Brazil, Rio de Janeiro State, Angra dos Reis Bay.

Holotype. $\widehat{o}$ CHIOC 35879 a.

Paratypes. CHIOC 35879 b (allotype +), 35880 ()), 35881 (ð), 35882 (ふ).

Remarks. Two paratypes males and one female deposited in the IPCAS collection.

Reference. Pereira et al. (2014b).

Neoascarophis Skrjabin, 1946

Neoascarophis mariae Pereira, Timi, Vieira \& Luque, 2012

Type host. Mullus argentinae Hubbs \& Marini, 1933 (Osteichthyes: Mullidae).

Infection site. Stomach and intestine.

Type locality. Brazil, Rio de Janeiro State, Rio de Janeiro (22 $\left.55^{\prime} \mathrm{S}, 43^{\circ} 12^{\prime} \mathrm{W}\right)$.

Holotype. $\widehat{O}$ CHIOC 35802 a.

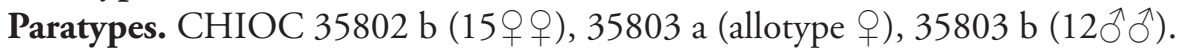

Remarks. Other paratypes deposited in the IPCAS collection.

Reference. Pereira et al. (2012a).

Pseudascarophis Ko, Margolis \& Machida, 1985

Pseudascarophis brasiliensis Pereira, Pereira, Timi \& Luque, 2013

Type host. Kyphosus sectatrix (Linnaeus, 1758) (Osteichthyes: Kyphosidae).

Infection site. Stomach.

Type locality. Brazil, Rio de Janeiro State, Angra dos Reis $\left(23^{\circ} 00^{\prime} \mathrm{S}, 44^{\circ} 10^{\prime} \mathrm{W}\right)$.

Holotype. $\lesssim$ CHIOC 35848 a.

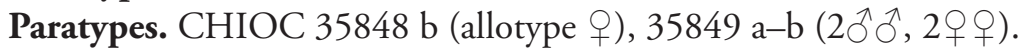

Reference. Pereira et al. (2013). 
Diplotriaenidae Skrjabin, 1916

Diplotriaena Railliet \& Henry, 1909

Diplotriaena zederi Pinto, Vicente \& Noronha, 1981

Type host. Xiphocolaptes albicollis (Vieillot, 1818) (Aves: Dendrocolaptidae).

Infection site. Body cavity.

Type locality. Brazil, Rio de Janeiro State, Angra dos Reis.

Holotype. $\lesssim$ CHIOC 31826 a.

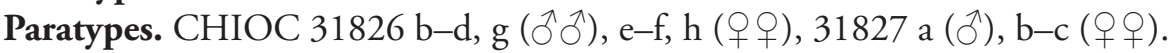

Reference. Pinto et al. (1981).

Dracunculidae Stiles, 1907

Dracunculus Reichard, 1759

Dracunculus brasiliensis Moravec \& Santos, 2009

Type host. Eunectes murinus (Linnaeus, 1758) (Serpentes: Boidae).

Infection site. Subcutaneous tissue (inside skin swelling) and body cavity (mesentery near lung).

Type locality. Brazil, Pará State, Mexiana Island (Amazon River delta), Santo Ambrósio Farm $\left(00^{\circ} 05^{\prime} 30^{\prime \prime S}, 49^{\circ} 34^{\prime} 50^{\prime \prime W}\right)$.

Holotype. $q$ CHIOC 35610 a.

Remarks. Paratype deposited in the IPCAS collection.

Reference. Moravec and Santos (2009).

Habronematidae Chitwood \& Wehr, 1932

Procyrnea Chabaud, 1975

Procyrnea anterovulvata Pinto, Vicente \& Noronha, 1996

Type host. Chelidoptera tenebrosa brasiliensis Sclater, 1862 (Aves: Bucconidae).

Infection site. Gizzard.

Type locality. Brazil, Espírito Santo State, Conceição da Barra.

Holotype. $\widehat{O}$ CHIOC 32783 a.

Paratypes. CHIOC 32783 b (ð), 32783 c, e-f (우 ㅇ), 32783 d (allotype +).

Reference. Pinto et al. (1996). 
Heligmonellidae Durette-Desset \& Chabaud, 1977

Freitastrongylus Gonçalves, Pinto \& Durette-Desset, 2007

*Freitastrongylus angelae Gonçalves, Pinto \& Durette-Desset, 2007

Type host. Dasyprocta fuliginosa Wagler, 1832 (Rodentia: Dasyproctidae).

Infection site. Stomach.

Type locality. Brazil, Amazonas State, Barcelos, Jauari waterway, left margin of the Aracá River.

Holotype. $₫$ CHIOC 35044 a.

Paratypes. CHIOC 34844, 35044 b (allotype + ), c- $\mathrm{f}($ ( + P), g-k (ふぶ), l-m (synlophe $\widehat{\partial} \hat{\jmath}), \mathrm{n}$ (synlophe).

Remarks. Dasyprocta leporina (Linnaeus, 1758) was cited as the type host in the original description, but $D$. fuliginosa is the host of all type material of CHIOC as indicated in the cataloguing data. Other male and female paratypes deposited in the MNHN collection.

Reference. Gonçalves et al. (2007).

Guerrerostrongylus Sutton \& Durette-Desset, 1991

Guerrerostrongylus gomesae Simóes, Santos \& Maldonado Jr., 2012

Type host. Oecomys mamorae (Thomas, 1906) (Rodentia: Cricetidae).

Infection site. Small intestine.

Type locality. Brazil, Mato Grosso do Sul State, Rio Negro Farm, Aquidauana (19³4'54"S, 56 $\left.14^{\circ} 62^{\prime \prime W}\right)$.

Holotype. $\sigma^{\wedge}$ CHIOC 37233.

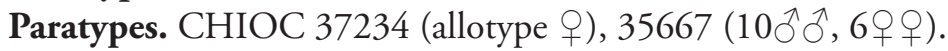

Reference. Simóes et al. (2012).

Guerrerostrongylus marginalis Weirich, Catzeflis \& Jiménez, 2016

Type host. Oecomys auyantepui Tate, 1939

Infection site. Small intestine.

Type locality. French Guiana, Municipality of Roura, Cacao $\left(04^{\circ} 33^{\prime} 708^{\prime \prime N}\right.$, $\left.52^{\circ} 26^{\prime} 590^{\prime \prime W}\right), 197 \mathrm{~m}$ high.

Paratypes. CHIOC 38104, 38105.

Remarks. Holotype, allotype and other paratypes deposited in the MNHN collection. Additional paratypes deposited in CNHE and HWML.

Reference. Weirich et al. (2016). 


\section{Hassalstrongylus Durette-Desset, 1971}

Hassalstrongylus luquei Costa, Maldonado Jr., Bóia, Lucio \& Simóes, 2014

Type host. Euryoryzomys russatus (Wagner, 1848) (Rodentia: Cricetidae).

Infection site. Small intestine.

Type locality. Brazil, Rio de Janeiro State, Angra dos Reis, Ilha Grande $\left(23^{\circ} 09^{\prime} 07.50^{\prime \prime} \mathrm{S}, 44^{\circ} 13^{\prime} 44.20^{\prime \prime} \mathrm{W}\right)$.

Holotype. $₫$ CHIOC 35928 a.

Paratypes. CHIOC 35928 b-c $(7 ð ð)$.

Reference. Costa et al. (2014).

Stilestrongylus Freitas, Lent \& Almeida, 1937

Stilestrongylus lanfrediae Souza, Digiani, Simóes, Luque, Rodrigues-Silva \& Maldonado Jr., 2009

Type host. Oligoryzomys nigripes

Infection site. Small intestine.

Type locality. Brazil, Rio de Janeiro State, Serra dos Órgãos, Teresópolis $\left(22^{\circ} 12^{\prime} 44^{\prime \prime} \mathrm{S}, 42^{\circ} 48^{\prime} 40^{\prime \prime} \mathrm{W}\right)$.

Holotype. ${ }^{\lambda}$ CHIOC 36925 a.

Paratypes. CHIOC 35536 (10今ึ, 10 우우), 36925 b (allotype ㅇ) ).

Reference. Souza et al. (2009).

Heterakidae Railliet \& Henry, 1912

Africana Travassos, 1920

Africana dardanelosi Ávila \& Silva, 2009

Type host. Hoplocercus spinosus Fitzinger, 1843 (Iguania: Hoplocercidae).

Infection site. Small and large intestine.

Type locality. Brazil, Mato Grosso State, Aripuanã $\left(10^{\circ} 9^{\prime} 0^{\prime \prime S}, 59^{\circ} 27^{\prime} 0^{\prime \prime W}\right)$.

Holotype. $\triangle$ CHIOC 35614 a.

Paratype. CHIOC 35614 b (allotype ?).

Remarks. Holotype and allotype cited as "CHIOC 3561" in the original description due to a mistake. Other paratypes deposited in CHIBB.

Reference. Ávila and Silva (2009). 
Heterakis Dujardin, 1845

Heterakis inglisi Vicente, Pinto \& Noronha, 1993

Type host. Crypturellus variegatus variegatus (Gmelin, 1789) (Aves: Tinamidae).

Infection site. Intestine.

Type locality. Brazil, Espírito Santo State.

Holotype. $\widehat{O}$ CHIOC 32850 a.

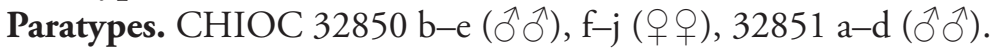

Reference. Vicente et al. (1993).

Hystrignathidae Travassos, 1919

Artigasia Christie, 1934

Artigasia milerai Morffe \& García, 2010

Type host. Passalus interstitialis Eschischoltz, 1829 (Coleoptera: Passalidae).

Infection site. Gut caeca.

Type locality. Cuba, La Habana province, Jaruco, Escaleras de Jaruco.

Paratypes. CHIOC 37823 (2웅).

Remarks. CHIOC number was not included in the original description. Holotype female and additional paratypes females deposited in CZACC.

Reference. Morffe and García (2010a).

Hystrignatus Leidy, 1850

Hystrignatus dearmasi Morffe \& García, 2010

Type host. Unidentified, short, blackish passalid beetle (Coleoptera: Passalidae).

Infection site. Gut caeca.

Type locality. Panama, Panama Province, Summit National Park.

Paratypes. CHIOC 37525 (2웅).

Remarks. CHIOC number was not included in the original description. Holotype female and additional paratypes females deposited in CZACC.

Reference. Morffe and García (2010b). 
Batwanema Morffe \& García, 2013

*Batwanema congo Morffe \& García, 2013

Type host. Pentalobus barbatus (Fabricius, 1801) (Coleoptera: Passalidae).

Infection site. Gut caeca.

Type locality. Democratic Republic of Congo, Ituri province, Mogwalu.

Paratype. CHIOC 37903 ().

Remarks. CHIOC number was not included in the original description. Holotype and paratype females deposited in CZACC. Other paratypes deposited in the RMCA collection.

Reference. Morffe and García (2013a).

Chokwenema Morffe \& García, 2013

*Chokwenema lepidophorum Morffe \& García, 2013

Type host. Didimoides cf. parastictus (Imhoff, 1843) (Coleoptera: Passalidae).

Infection site. Gut caeca.

Type locality. Democratic Republic of Congo, Ituri province, Mogwalu.

Paratype. CHIOC 37904 ( + ).

Remarks. CHIOC number was not included in the original description. Holotype and paratype females deposited in CZACC. Other paratypes deposited in the RMCA collection.

Reference. Morffe and García (2013a).

Kongonema Morffe \& García, 2013

*Kongonema meyeri Morffe \& García, 2013

Type host. Didimus sp. (Coleoptera: Passalidae).

Infection site. Gut caeca.

Type locality. Democratic Republic of Congo, Kivu Region, Katale $\left(01^{\circ} 19^{\prime} \mathrm{S}\right.$, $\left.29^{\circ} 22^{\prime} \mathrm{E}\right)$.

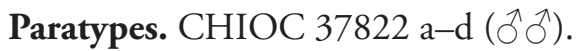

Remarks. CHIOC number was not included in the original description. Female holotype and male and female paratypes deposited in CZACC. Other paratypes deposited in the RMCA collection.

Reference. Morffe and García (2013b). 
Metastrongylidae Leiper, 1908

Angiostrongylus Kamensky, 1905

Angiostrongylus felineus Vieira, Muniz-Pereira, Lima, Moraes Neto, Guimarães \& Luque, 2013

Type host. Puma yagouaroundi (Geoffroy, 1803) (Carnivora: Felidae).

Infection site. Pulmonary arteries.

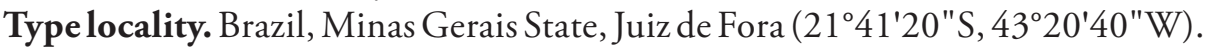

Holotype. $\delta$ CHIOC 35812 a.

Paratypes. CHIOC 35812 b (allotype + ), $35812 \mathrm{c}-\mathrm{d}(\AA$, $q)$.

Reference. Vieira et al. (2013).

Molineidae Durette-Desset \& Chabaud, 1977

Hadrostrongylus Hoppe \& Nascimento, 2007

${ }^{*}$ Hadrostrongylus speciosum Hoppe \& Nascimento, 2007

Type host. Dasypus novemcinctus

Infection site. Mucosa and lumen of the cecum and colon.

Type locality. Brazil, Mato Grosso do Sul State, Aquidauana.

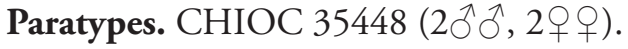

Remarks. Male holotype and female allotype deposited in the FCAV/UNESP helminthological collection of Jaboticabal, São Paulo State.

Reference. Hoppe and Nascimento (2007).

Oswaldocruzia Travassos, 1917

Oswaldocruzia belenensis Santos, Geise, Maldonado \& Lanfredi, 2008

Type host. Rhinella marina (Linnaeus, 1758) [= Chaunus marinus] (Amphibia: Bufonidae).

Infection site. Small intestine.

Type locality. Brazil, Pará State, Belém (01²8'03"S, 48²0'18"W).

Holotype. CHIOC 36855 a.

Paratypes. CHIOC 36855 b (allotype ㅇ), 36855 c, f (웅), d-e (ふ̋̂)).

Reference. Santos et al. (2008b). 
Schulzia Travassos, 1937

Schulzia usu Lent \& Santos, 1989

Type host. Atelopus oxyrhynchus

Infection site. Small intestine.

Type locality. Venezuela, Mérida.

Holotype $\delta$ and allotype + . CHIOC 31668.

Paratype. CHIOC 32355.

Reference. Lent and Santos (1989).

Onchocercidae Leiper, 1911

Cercopithifilaria Eberhard, 1980

Cercopithifilaria bainae Almeida \& Vicente, 1984

Type host. Canis familiaris Linnaeus, 1758 (Carnivora: Canidae).

Infection site. Subcutaneous and intramuscular tissue.

Type locality. Brazil, Rio de Janeiro State, Rio de Janeiro.

Holotype. $\lesssim$ CHIOC 32176 a.

Paratype. CHIOC 32176 b (allotype 9 ).

Reference. Almeida and Vicente (1984).

Litomosoides Chandler, 1931

Litomosoides chagasfilhoi Moraes Neto, Lanfredi \& Souza, 1997

Type host. Akodon cursor (Winge, 1887) (Rodentia: Muridae).

Infection site. Abdominal cavity.

Type locality. Brazil, Rio de Janeiro State, Rio Bonito, Catimbau Grande (2242'30"S, 42³7'34"W).

Holotype. ô CHIOC 33529.

Paratype. CHIOC 33530 (allotype +).

Remarks. Other paratypes deposited in the MNHN collection.

Reference. Moraes Neto et al. (1997).

Litomosoides galizai Bain, Petit \& Diagne, 1989

Type host. Oecomys trinitatis (Allen \& Chapman, 1893)

Infection site. Body cavity and lung.

Type locality. Brazil, Pará State, Carajás. 
Paratypes. CHIOC 32643 (2q $q$ ).

Remarks. Holotype and allotype deposited in the MNHN collection. CHIOC number was not included in the original description.

Reference. Bain et al. (1989).

\section{Litomosoides kohnae Bain, Petit \& Diagne, 1989}

Type host. Nectomys squamipes

Infection site. Body cavity and lung.

Type locality. Brazil, São Paulo State, São Paulo.

Holotype. $q$ CHIOC 31056 c.

Paratypes. CHIOC 31056 a (đ), 31056 b (allotype đ), 32642 (ㅇ , = 31056 d).

Remarks. The slide CHIOC $31056 \mathrm{~d}$ was dismounted and received the number 32642.

Reference. Bain et al. (1989).

\section{Ornithofilaria Gonnert, 1937}

Ornithofilaria pitangi Vicente, Pinto \& Noronha, 1980

Type host. Megarynchus pitangua (Linnaeus, 1766) (Aves: Tyrannidae).

Infection site. Infraorbital sinus.

Type locality. Brazil, Rio de Janeiro State, Angra dos Reis.

Holotype. $\widehat{o}$ CHIOC 31782 a.

Paratypes. CHIOC 31782 b-e (ふぶ), f-j (우).

Reference. Vicente et al. (1980).

Oxyuridae Cobbold, 1864

Caroloxyuris Jiménez-Ruiz \& Gardner, 2003

${ }^{*}$ Caroloxyuris boliviensis Jiménez-Ruiz \& Gardner, 2003

Type host. Oxymycterus paramensis Thomas, 1902 (Rodentia: Cricetidae).

Infection site. Cecum and large intestine.

Type locality. Bolivia, Chuquisaca Department, $2 \mathrm{~km} \mathrm{SW}$ of Monteagudo (1949'S, 635' $\mathrm{W}), 1130 \mathrm{~m}$ high.

Paratype. CHIOC 34809.

Remarks. Paratype from CHIOC collected in Cochabamba, $13 \mathrm{~km} \mathrm{~N}$ of Colomi (17 $\left.13^{\prime} 29^{\prime \prime S}, 65^{\circ} 53 " 30 " \mathrm{~W}\right), 3152 \mathrm{~m}$ high. Holotype, allotype, and paratypes deposited in the HWML collection. Other two paratypes deposited in CNHE.

Reference. Jiménez-Ruiz and Gardner (2003). 
Gracilioxyuris Feijó, Lopes Torres, Maldonado Jr. \& Lanfredi, 2008

*Gracilioxyuris agilis Feijó, Torres, Maldonado Jr. \& Lanfredi, 2008

Type host. Gracilinanus agilis (Burmeister, 1854) (Didelphimorphia: Didelphidae).

Infection site. Cecum.

Type locality. Brazil, Mato Grosso do Sul State, Pantanal, Nhumirim Farm (18 $\left.59^{\circ} 00^{\prime \prime S}, 56^{\circ} 39^{\prime} 00^{\prime \prime W}\right)$.

Holotype. $\bigcirc 35518$ a.

Paratypes. CHIOC 35517 (allotype 9), 35518 b.

Remarks. CHIOC 35517 was collected in the Rio Negro Farm, Nhecolândia Region (19³4'54"S, 56 14'62"W), Pantanal, Mato Grosso do Sul State.

Reference. Feijó et al. (2008).

Pharyngodonidae Travassos, 1919

Brasilnema Moravec, Kohn \& Fernandes, 1992

*Brasilnema pimelodellae Moravec, Kohn \& Fernandes, 1992

Type host. Pimelodella lateristriga

Infection site. Intestine.

Type locality. Brazil, Paraná State, Paraná River, Guaíra.

Paratypes. CHIOC 32718 a-b, e (ふ̋̂), c-d, f-i (우), 32719 a-d (우).

Remarks. Holotype, allotype, and other paratypes deposited in the IPCAS collection. Additional paratypes deposited in MNHN.

Reference. Moravec et al. (1992a).

Ichthyouris Inglis, 1962

Ichthyouris brasiliensis Moravec, Kohn \& Fernandes, 1992

Type host. Megalancistrus parananus (Peters, 1881) (Osteichthyes: Locariidae).

Infection site. Intestine.

Type locality. Brazil, Paraná State, Paraná River, Guaíra.

Paratypes. CHIOC 32649, $32721 \mathrm{a}-\mathrm{b}(\hat{\jmath}$,, ).

Remarks. Holotype, allotype, and other paratypes deposited in the IPCAS collection. Additional paratypes deposited in MNHN.

Reference. Moravec et al. (1992a). 
Ichthyouris laterifilamenta Moravec, Kohn \& Fernandes, 1992

Type host. Trachydoras paraguayensis (Eigenmann \& Ward, 1907) (Osteichthyes: Doradidae).

Infection site. Intestine.

Type locality. Brazil, Paraná State, Foz do Iguaçú, Reservoir of the hydroelectric power of Itaipú $\left(24^{\circ} 20^{\prime} \mathrm{S}, 52^{\circ} 38^{\prime} \mathrm{W}\right)$.

Paratype. CHIOC 32925.

Remarks. Holotype, allotype, and other paratypes deposited in the IPCAS collection.

Reference. Moravec et al. (1992b).

Ichthyouris voltagrandensis Martins, Yoshitoshi \& Umekita, 2001

Type host. Myleus tiete (Eigenmann \& Norris, 1900) (Osteichthyes: Serrasalmidae).

Infection site. Intestine.

Type locality. Brazil, Minas Gerais State, Volta Grande Reservoir.

Holotype. ô CHIOC 33852 a.

Paratypes. CHIOC 33852 b (allotype + ), 33852 c $\left(5 \jmath^{\Uparrow}\right), 33852 \mathrm{~d}(7$ 우 + ).

Reference. Martins et al. (2001).

Parasynodontisia Moravec, Kohn \& Fernandes, 1992

*Parasynodontisia petterae Moravec, Kohn \& Fernandes, 1992

Type host. Rhinelepis aspera Spix \& Agassiz, 1829 (Osteichthyes: Locariidae).

Infection site. Intestine.

Type locality. Brazil, Paraná State, Paraná River, Guaíra.

Paratypes. CHIOC 32652, 32720 a ( $\left.O^{\Uparrow}\right), \mathrm{b}($ ( + ).

Remarks. Holotype, allotype, and other paratypes deposited in the IPCAS collection. Additional paratypes deposited in MNHN.

Reference. Moravec et al. (1992b).

Skrjabinodon Inglis, 1968

Skrjabinodon heliocostai Vicente, Vrcibradic, Muniz-Pereira \& Pinto, 2000

Type host. Notomabuya frenata (Cope, 1862) [= Mabuya frenata] (Autarchoglossa: Scincidae). 
Infection site. Large intestine.

Type locality. Brazil, São Paulo State, Valinhos.

Holotype. ते CHIOC 33965 a.

Paratypes. CHIOC 33965 b (allotype + ), CHIOC 33965 c-e (ふぶ), f-h (

Reference. Vicente et al. (2000b).

Skrjabinodon spinosulus Vicente, Vrcibradic, Rocha \& Pinto, 2002

Type host. Aspronema dorsivittatum (Cope, 1862) [= Mabuya dorsivittata] (Autarchoglossa: Scincidae).

Infection site. Large and small intestine.

Type locality. Brazil, Rio de Janeiro State, National Park of Itatiaia, Prateleiras.

Holotype. $q$ CHIOC 34539 a.

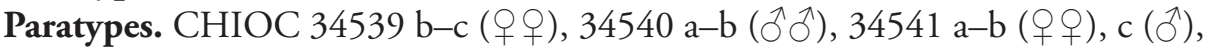
$\mathrm{d}(2$ 우), $\mathrm{e}-\mathrm{f}$ (larvae).

Remarks. Paratypes CHIOC 34541 a-f collected in the Ecological Station of Itirapina, São Paulo State.

Reference. Vicente et al. (2002).

Philometridae Baylis \& Daubney, 1926

Philometra Costa, 1845

Philometra nattereri Cárdenas, Moravec, Fernandes \& Morais, 2012

Type host. Pygocentrus nattereri Kner, 1858 (Osteichthyes: Serrasalmidae).

Infection site. Oculo-orbits and nasal mucosa.

Type locality. Brazil, Amazonas State, Maracá Lake (0350'32.8"S, 62³4'32.4"W), Coari.

Holotype. + CHIOC 35777.

Paratypes. CHIOC 35778 (2q $q$ ), 35779 (), 35780 (q), 35781 (q), 35782.

Remarks. CHIOC 35779 collected in the Baixio Lake $\left(03^{\circ} 17^{\prime} 27.2^{\prime \prime} \mathrm{S}\right.$, $\left.60^{\circ} 04^{\prime} 29.6^{\prime \prime} \mathrm{W}\right)$, Iranduba; CHIOC 35780 collected in the Iauara Lake $\left(03^{\circ} 36^{\prime} 39.2^{\prime \prime} \mathrm{S}\right.$, 61 ${ }^{\circ} 16^{\prime} 33.0^{\prime \prime W), ~ M a n a c a p u r u ; ~ C H I O C ~} 35781$ collected in the Araçá Lake (034' $\left.15.8^{\prime \prime} \mathrm{S}, 62^{\circ} 20^{\prime} 10.3^{\prime \prime W}\right)$, Codajás; CHIOC 35782 collected in the Ananá Lake (03053'54.8"S, 61 $\left.40^{\circ} 18.4 \mathrm{WW}\right)$, Anori (all localities in the Amazonas State). Other paratypes deposited in the INPA collection.

Reference. Cárdenas et al. (2012). 
Physalopteridae Railliet, 1893

Physaloptera Rudolphi, 1819

Physaloptera bainae Pereira, Alves, Rocha, Souza Lima \& Luque, 2014

Type host. Salvator merianae (Duméril \& Bibron, 1839) (Autarchoglossa: Teiidae).

Infection site. Stomach.

Type locality. Brazil, Minas Gerais State, Juiz de Fora, Parque da Lajinha (21ํㄱ' $32^{\prime \prime}$ S, $\left.43^{\circ} 22^{\prime} 6^{\prime \prime W}\right)$.

Holotype. $\overbrace{}^{\lambda}$ CHIOC 35885 a.

Paratypes. CHIOC 35885 b (allotype 우), 35885 c (đ̂), 35885 d (2웅).

Reference. Pereira et al. (2014b).

Physaloptera galvaoi Sáo Luiz, Simóes, Lopes Torres, Barbosa, Santos, Giese, Rocha \& Maldonado Jr., 2015

Type host. Cerradomys subflavus (Wagner, 1842) (Rodentia: Cricetidae).

Infection site. Stomach.

Type locality. Brazil, Minas Gerais State, São Roque de Minas, Serra da Canastra National Park $\left(20^{\circ} 13^{\prime} 28.30^{\prime \prime}\right.$ S, 4630'39.20"W).

Holotype. $₫$ CHIOC 36758 a.

Paratype. CHIOC 36758 b (allotype + ).

Reference. São Luiz et al. (2015).

Physaloptera herthameyerae Lopes Torres, Maldonado Jr. \& Lanfredi, 2009

Type host. Gracilinanus agilis

Infection site. Stomach.

Type locality. Brazil, Mato Grosso do Sul State, Pantanal, Alegria Farm $\left(19^{\circ} 15^{\prime} 01^{\prime \prime S}\right.$, $\left.57^{\circ} 01^{\prime} 29^{\prime \prime} \mathrm{W}\right)$.

Holotype. $\stackrel{\AA}{1} \mathrm{CHIOC} 35651$ a.

Paratypes. CHIOC 35651 b (đ), 35652 a (allotype + ), 36652 b (q).

Reference. Lopes Torres et al. (2009).

Physaloptera tupinambae Pereira, Alves, Rocha, Lima \& Luque, 2012

Type host. Salvator merianae [= Tupinambis merianae]

Infection site. Stomach.

Type locality. Brazil, Minas Gerais State, Juiz de Fora.

Holotype. ${ }^{\top}$ CHIOC 35811 a. 


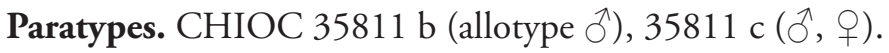

Reference. Pereira et al. (2012b).

Quimperiidae Gendre, 1928

Neoparaseuratum Moravec, Kohn \& Fernandes, 1992

*Neoparaseuratum travassosi Moravec, Kohn \& Fernandes, 1992

Type host. Pterodoras granulosus

Infection site. Intestine.

Type locality. Brazil, Paraná State, Paraná River near Guaíra.

Holotype. $\widehat{O}$ CHIOC 32722 a.

Paratype. CHIOC 32722 b (allotype + ).

Remarks. There is no paratype CHIOC 32722 "c" as indicated in the original description, which was a mistake. Other paratype deposited in the IPCAS collection.

Reference. Moravec et al. (1992c).

Raphidascarididae Hartwich, 1954

Hysterothylacium Ward \& Magath, 1917

Hysterothylacium deardorffoverstreetorum Knoff, Felizardo, Ińiguez, Maldonado Jr., Torres, Pinto \& Gomes, 2012

Type host. Paralichthys isosceles Jordan, 1891 (Osteichthyes: Paralichthyidae).

Infection site. Abdominal cavity, abdominal musculature, stomach, stomach mucosa, mesentery, intestine, heart serosa, kidney serosa, liver serosa, ovary, ovary serosa, and spleen serosa.

Type locality. Brazil, Rio de Janeiro State, Angra dos Reis $\left(21^{\circ} 15^{\prime}-23^{\circ} 23^{\prime} \mathrm{S}\right.$, $\left.40^{\circ} 29^{\prime}-44^{\circ} 28^{\prime} \mathrm{W}\right)$.

Holotype. CHIOC 37523 a (L3 larvae).

Paratypes. CHIOC 37523 b-e, 35771 (L3 larvae).

Reference. Knoff et al. (2012).

Rhabdiasidae Railliet, 1916

Rhabdias Stilles \& Hassall, 1905

Rhabdias filicaudalis Barrella, Santos \& Silva, 2010

Type host. Spilotes pullatus Linnaeus, 1758 (Serpentes: Colubridae).

Infection site. Lungs.

Type locality. Brazil, São Paulo State, Avaré ( $\left.23^{\circ} 6^{\prime} \mathrm{S}, 48^{\circ} 55^{\prime} \mathrm{W}\right)$.

Holotype. + CHIOC 35653 a. 
Paratypes. CHIOC 35653 b (5우우).

Remarks. Other paratypes deposited in CHIBB.

Reference. Barella et al. (2010).

Rhabdias paraensis Santos, Melo, Silva, Giese \& Furtado, 2011

Type host. Rhinella marina

Infection site. Lungs.

Type locality. Brazil, Pará State, Belém (01²8'03"S, 48²0'18"W).

Holotype. Hermaphrodite $q$ CHIOC 35705 a.

Paratypes. CHIOC 35705 b (ten paratypes).

Reference. Santos et al. (2011).

Serpentirhabdias Trach, Kuzmin \& Snyder, 2014

Serpentirhabdias viperidicus Morais, Aguiar, Müller, Narciso, Silva \& Silva, 2016

Type host. Bothrops moojeni Hoge, 1966

Infection site. Lungs.

Type locality. Brazil, São Paulo State, Castilho, Private Reserve of Natural Heritage 'Foz do Rio Aguapeî' (2103'04.9"S, 51 52 '52.6"W).

Holotype. CHIOC 38318 a.

Paratype. CHIOC 38318 b.

Remarks. Other paratypes deposited in CHIBB.

Reference. Morais et al. (2016).

Rictulariidae Hall, 1915

Pterygodermatites (Multipectines) Quentin, 1969

Pterygodermatites (Multipectines) pluripectinata Hoppe, Araújo, Tebaldi \& Nascimento, 2010

Type host. Cerdocyon thous

Infection site. Small intestine.

Type locality. Brazil, Paraíba State, Patos (0646'19"-07³8'52"S, 36²2'52"$\left.38^{\circ} 08^{\prime} 56^{\prime \prime} \mathrm{W}\right)$.

Paratypes. CHIOC 35628 (ô, ९).

Remarks. Paratype from CHIOC cited as "35525" in the original description due to a mistake. Holotype and allotype deposited in the FCAV/UNESP of Jaboticabal, São Paulo State.

Reference. Hoppe et al. (2010). 
Pterygodermatites (Paucipectines) Quentin, 1969

Pterygodermatites (Paucipectines) andyraicola Cardia, Tebaldi, Fornazari, Menozzi, Langoni, Nascimento, Bresciani \& Hoppe, 2015

Type host. Eumops glaucinus (Wagner, 1843) (Chiroptera: Molossidae).

Infection site. Mucosa of the small intestine.

Type locality. Brazil, São Paulo State, Botucatú (2252'47"S, 48²6'42"W).

Paratypes. CHIOC 35826 ( $\precsim$, ㅇ).

Remarks. Holotype, allotype and additional paratypes deposited in FCAV/UNESP of Araçatuba, São Paulo State. Paratypes from CHIOC cited as "35824" in the original description due to a mistake. CHIOC 35826 collected in Jaú $\left(22^{\circ} 17^{\prime} 44^{\prime \prime} S\right.$, $48^{\circ} 33^{\prime} 28^{\prime \prime}$ W), São Paulo State.

Reference. Cardia et al. (2015).

Strongyloididae Chitwood \& McIntosh, 1934

Strongyloides Grass, 1879

Strongyloides ferrerai Rodrigues, Vicente \& Gomes, 1985

Type host. Kerodon rupestris (Wied-Neuwied, 1820) (Rodentia: Caviidae).

Infection site. Small intestine.

Type locality. Brazil, Piauí State, Floriano Peixoto.

Holotype. + CHIOC 32172 a.

Paratypes. CHIOC 32172 b-e (우).

Reference. Rodrigues et al. (1985).

Subuluridae Travassos, 1914

Subulura Molin, 1860

Subulura lacertilia Vicente, Van Sluys, Fontes \& Kiefer, 2000

Type host. Eurolophosaurus nanuzae (Rodrigues, 1981) [= Tropidurus nanuzae] (Iguania: Tropiduridae).

Infection site. Large and small intestine.

Type locality. Brazil, Minas Gerais State, Serra do Cipó (19²0'S, 434ㄴ'W).

Holotype. $\widehat{O}$ CHIOC 34196 a.

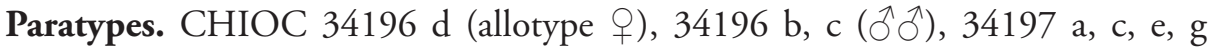

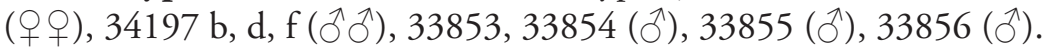

Reference. Vicente et al. (2000a). 
Tetrameridae Travassos, 1914

Synhimantus (Synhimantus) Railliet, Henry \& Sisoff, 1912

Synhimantus (Synhimantus) magnipapillatus Vicente, Pinto e Noronha, 1996

Type host. Nyctanassa violacea cayennensis (Gmelin, 1789) (Aves: Ardeidae).

Infection site. Gizzard.

Type locality. Brazil, Rio de Janeiro State, Rio de Janeiro.

Holotype. $\lesssim$ CHIOC 33182 a.

Paratypes. CHIOC $33182 \mathrm{~b}, \mathrm{f}-\mathrm{h}($ ふぇ), c-e (우).

Reference. Vicente et al. (1996).

Tetrameres (Tetrameres) Creplin, 1846

Tetrameres (Tetrameres) spirospiculum Pinto \& Vicente, 1995

Type host. Theristicus caudatus caudatus (Boddaert, 1783) (Aves: Threskiornithidae).

Infection site. Gizzard (females in proventricular glands, males free in the lumen).

Type locality. Brazil, Mato Grosso do Sul State, Salobra.

Holotype. $\stackrel{\jmath}{ }$ CHIOC 33173 a.

Paratypes. CHIOC 33173 b-c $(ð ð), 33186$ c (allotype 우).

Reference. Pinto and Vicente (1995).

Trichostrongylidae Witenberg, 1925

Hoazinstrongylus Pinto \& Gomes, 1985

${ }^{*}$ Hoazinstrongylus amazonensis Pinto \& Gomes, 1985

Type host. Opisthocomus hoazin (Müller, 1776) (Aves: Opisthocomidae).

Infection site. Proventriculus and gizzard.

Type locality. Brazil, Amazonas State, Paraná de Cambixa, Careiro Island.

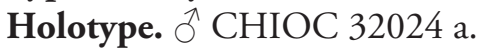

Paratypes. CHIOC $32024 \mathrm{~b}-\mathrm{e}$ (우 우), $32025 \mathrm{a}-\mathrm{b}$ (우 우), $32026 \mathrm{a}-\mathrm{b}\left({ }^{\lambda}{ }^{\Uparrow}\right), \mathrm{c}-\mathrm{d}$ (우우).

Reference. Pinto and Gomes (1985). 
Viannaiidae Durette-Desset \& Chabaud, 1981

Avellaria Freitas \& Lent, 1934

Avellaria intermedia Durette-Desset, Gonçalves \& Pinto, 2006

Type host. Dasyprocta fuliginosa

Infection site. Small intestine.

Type locality. Brazil, Amazonas State, Barcelos, Jauari waterway, left margin of the Aracá River, Três Barracas settlement (0 ${ }^{\circ} 58^{\prime} 29^{\prime \prime}$ S, 62 $\left.{ }^{\circ} 55^{\prime} 27^{\prime \prime} \mathrm{W}\right)$.

Holotype ${ }^{\hat{A}}$ and allotype + . CHIOC 35416.

Paratypes. CHIOC 34883, $35072 \mathrm{a}-\mathrm{g}, 35417$ (6へぇ), 35418 (7우우).

Reference. Durette-Desset et al. (2006).

Vianella Neveu-Lemaire, 1934

Vianella trichospicula Durette-Desset, Gonçalves \& Pinto, 2006

Type host. Dasyprocta fuliginosa

Infection site. Small intestine.

Type locality. Brazil, Amazonas State, Barcelos, Jauari waterway, left margin of the Aracá River, Três Barracas settlement (058'29"S, 6255'27"W).

Holotype $\delta$ and allotype $O$. CHIOC 35419.

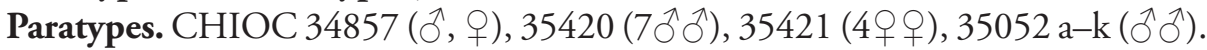

Reference. Durette-Desset et al. (2006).

Xustrostomatidae Hunt, 2002

Trachyglossoides García \& Morffe, 2015

*Trachyglossoides jimenoi García \& Morffe, 2015

Type host. Spirobollelus sp. (Diplopoda: Spirobolellidae).

Infection site. Hind gut.

Type locality. Cuba, Mayabeque Province, San José de las Lajas, La Jaula.

Paratypes. CHIOC 38211 a ( $($ ), 38211 b (ठ̊).

Remarks. CHIOC number was not included in the original description. Male holotype and male and female paratypes deposited in CZACC. Other paratypes deposited in the RIT collection.

Reference. García and Morffe (2015). 
Phylum Cnidaria Hatschek, 1888

Unranked subphylum Myxozoa Grassé, 1970

Class Myxosporea Bütschli, 1881

Order Bivalvulida Shulman, 1959

Myxobolidae Thélohan, 1892

Henneguya Thélohan, 1892

Henneguya garavelli Martins \& Onaka, 2006

Type host. Cyphocharax nagelii (Steindachner, 1881) (Osteichthyes: Curimatidae).

Infection site. Gill filaments.

Type locality. Brazil, São Paulo State, São José do Rio Pardo, Rio do Peixe Reservoir.

Holotype. CHIOC 34986.

Paratype. CHIOC 34818 (fixed gills).

Remarks. CHIOC samples cited as "specimens deposited" in the original description. No additional type material is deposited in other collections.

Reference. Martins and Onaka (2006).

Myxobolus Bütschli, 1882

Myxobolus peculiaris Martins \& Onaka, 2006

Type host. Cyphocharax nagelii

Infection site. Gill filaments.

Type locality. Brazil, São Paulo State, São José do Rio Pardo, Rio do Peixe Reservoir.

Holotype. CHIOC 34987.

Paratype. CHIOC 34834 (fixed gills).

Remarks. CHIOC samples cited as "specimens deposited" in the original description. No additional type material is deposited in other collections.

Reference. Martins and Onaka (2006).

Phylum Annelida Lamarck, 1809

Class Polychaeta Grube, 1850

Order Eunicida Dales, 1962

Histriobdellidae Vaillant, 1890

Stratiodrilus Haswell, 1900

Stratiodrilus vilae Amato, 2001

Type host. Parastacus brasiliensis (von Martens, 1869) (Decapoda: Parastacidae).

Infection site. Branchial chamber. 
Type locality. Brazil, Rio Grande do Sul State, Taquara (29 $\left.37^{\prime} \mathrm{S}, 50^{\circ} 47^{\prime} \mathrm{W}\right)$. Holotype. $q$ CHIOC 34334 a.

Paratypes. CHIOC 34334 b (allotype đ̂), 34335 a-b, e (우), c-d, f (ðへ).

Remarks. Other paratypes deposited in the USNM collection.

Reference. Amato (2001).

Class Hirudinea Linnaeus, 1758

Order Rhynchobdellida (Blanchard, 1894)

Ozobranchidae (Pinto, 1921)

Unoculubranchiobdella Peralta, Matos \& Serra-Freire, 1998

*Unoculubranchiobdella expansa Peralta, Matos \& Serra-Freire, 1998

Type host. Podocnemis expansa (Schweigger, 1812) (Testudines: Podocnemididae).

Infection site. Carapace, plastron, head, over the eyes, pelvic appendages, cloaca, mouth and nostrils.

Type locality. Brazil, Pará State, Belém, Zoobotanical Park of the Emílio Goeldi Museum.

Holotype. CHIOC 37524 a.

Paratypes. CHIOC 33598, 37524 b-e.

Remarks. The holotype and some paratypes received new numbers because part of the type material was mounted from CHIOC 33598 by the authors after the publication. CHIOC 33598 was indicated as holotype and paratypes in the original description.

Reference. Peralta et al. (1998).

Phylum Arthropoda von Siebold, 1848

Class Maxillopoda Dahl, 1956

Subclass Copepoda Milne Edwards, 1840

Order Poecilostomatoida Thorell, 1859

Bomolochidae Sumpf, 1871

Hamaticolax Ho \& Lin, 2006

Hamaticolax unisagittatus (Tavares \& Luque, 2003)

Type host. Centropomus undecimalis (Block, 1792) (Osteichthyes: Centropomidae).

Infection site. Gills.

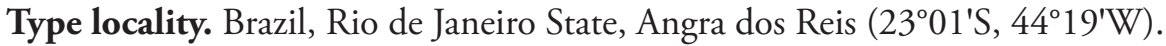

Paratypes. CHIOC 34819 (10우 $)$.

Remarks. Species placed in Acantholochus Cressey, 1984 by Tavares and Luque (2003) and transferred to Hamaticolax by Morales-Serna and Gómez (2010). Holotype and other female paratypes deposited in the MNRJ carcinological collection.

References. Tavares and Luque (2003), Morales-Serna and Gómez (2010). 
Chondracanthidae Milne Edwards, 1840

Pseudolernentoma Luque \& Alves, 2003

\section{*Pseudolernentoma brasiliensis Luque \& Alves, 2003}

Type host. Genypterus brasiliensis Regan, 1903 (Osteichthyes: Ophidiidae).

Infection site. Oral cavity.

Type locality. Brazil, coastal zone of the Rio de Janeiro State $\left(21-23^{\circ} \mathrm{S}, 41-45^{\circ} \mathrm{W}\right)$.

Paratypes. CHIOC 34892 (5우), 34893 (3ठึ

Remarks. Female holotype, male allotype, and other male and female paratypes deposited in the MNRJ carcinological collection.

Reference. Luque and Alves (2003).

Ergasilidae von Nordmann, 1832

Brasergasilus Thatcher \& Boeger, 1983

Brasergasilus bifurcatus Santos, Thatcher \& Brasil-Sato, 2007

Type host. Pygocentrus piraya (Cuvier, 1819)

Infection site. Gill filaments and nasal fossae.

Type locality. Brazil, Minas Gerais State, Upper São Francisco River, Três Marias Reservoir (18¹2'59"S, 4517'34"W).

Holotype. + CHIOC 36841.

Paratypes. CHIOC 35502 ()), 35503 ()), 35504 (q), 36842 (q), 36843 (q), $36844($ ( $)$.

Remarks. CHIOC 35504, 36843, and 36844 collected from the serrasalmid fish Serrasalmus brandtii Lütken, 1875.

Reference. Santos et al. (2007).

Ergasilus von Nordmann, 1832

Ergasilus thatcheri Engers, Boeger \& Brandáo, 2000

Type host. Rhamdia quelen (Quoy \& Gaimard, 1824) (Osteichthyes: Heptapteridae). Infection site. Gills filaments.

Type locality. Brazil, Rio Grande do Sul State, Barragem do Capané, Cachoeira do Sul. Holotype. + CHIOC 34008 a.

Paratypes. CHIOC $33840 \mathrm{a}-\mathrm{c}, 34008 \mathrm{~b}-\mathrm{f}$ (우우).

Remarks. Other paratypes deposited in the collections of HWML and USNM.

Reference. Engers et al. (2000). 


\section{Ergasilus youngi Tavares \& Luque, 2005}

Type host. Aspistor luniscutis (Valenciennes, 1840) (Osteichthyes: Ariidae).

Infection site. Gills.

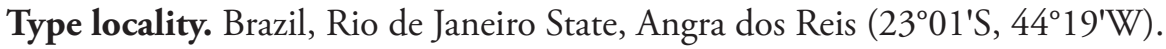

Holotype. + CHIOC 35393.

Paratypes. CHIOC 35392 (5우), 35394 (5우).

Reference. Tavares and Luque (2005).

\section{Acknowledgments}

The authors are grateful to Erik Russell Wild for reviewing the English of this manuscript. We thank Adriana Mainenti, Juliane Nunes Bernardo, Magda Sanches, Marianna Silva Costa, and Tainah Domingos Soares for technical support.

\section{References}

Almeida GLG, Vicente JJ (1984) Cercopithifilaria bainae sp. n. parasita de Canis familiaris (L.) (Nematoda, Filarioidea). Atas da Sociedade de Biologia do Rio de Janeiro 24: 18.

Amato JFR (2001) A new species of Stratiodrilus (Polychaeta, Histriobdellidae) from freshwater crayfishes of southern Brazil. Iheringia, Série Zoologia 90:37-44. https://doi.org/10.1590/ S0073-47212001000100004

Amin OM (1985) Classification. In: Crompton DWT, Nickol BB (Eds) Biology of the Acanthocephala. Cambridge University Press, London and New York, 27-72.

Amin OM (2013) Classification of the Acanthocephala. Folia Parasitologica 60(4): 273-305. https://doi.org/10.14411/fp.2013.031

Ávila RW, Silva RJ (2009) A new species of Africana (Nematoda: Heterakidae) from lizards of Southern Amazon, Brazil. Journal of Parasitology 95(5): 1156-1158. https://doi. org/10.1645/GE-1949.1

Ávila RW, Strüssmann C, Silva RJ (2010) A new species of Cosmocercoides (Nematoda: Cosmocercidae) from a gymnophthalmid lizard of western Brazil. Journal of Parasitology 96 (3): 558-560. https://doi.org/10.1645/GE-2336.1

Bain O, Petit G, Diagne M (1989) A morphological-study of some Litomosoides species from rodents; taxonomic conclusions. Annales de Parasitologie Humaine et Comparee 64(4): 268-289. https://doi.org/10.1051/parasite/1989644268

Barrella TH, Santos KR, Silva RJ (2010) Rhabdias filicaudalis n. sp. (Nematoda: Rhabdiasidae) from the snake Spilotes pullatus (Serpentes: Colubridae) in Brazil. Journal of Helminthology 84(3): 292-296. https://doi.org/10.1017/S0022149X09990691

Beesly PL, Ross GJB, Glasby CJ (Eds) (2000) Polychaetes and Allies: The Southern Synthesis. Fauna of Australia. Vol. 4A. Polychaeta, Myzostomida, Pogonophora, Echiura, Sipuncula. CSIRO Publishing, Melbourne, 465 pp. 
Boucher S (2005) Évolution et phylogénie des Coléoptères Passalidae (Scarabaeoidea). Annales de la Société entomologique de France 41(3-4): 239-604. https://doi.org/10.1080/0037 9271.2005.10697444

Braicovich PE, Lanfranchi AL, Farber MD, Marvaldi AE, Luque JL, Timi JT (2014) Genetic and morphological evidence reveals the existence of a new family, genus and species of Echinorhynchida (Acanthocephala). Folia Parasitologica 61(4): 377-384. https://doi. org/10.14411/fp.2014.044

Brasil-Sato MC, Pavanelli GC (1998) Neochinorhynchus pimelodisp. n. (Eoacanthocephala, Neoechinorhynchidae) parasitizing Pimelodus maculatus Lacépède, "mandi-amarelo" (Siluroidei, Pimelodidae) from the basin of the São Francisco river, Três Marias, Minas Gerais, Brazil. Revista Brasileira de Zoologia 15(4): 1003-1011. https://doi.org/10.1590/S0101-81751998000400019

Cárdenas MQ, Moravec F, Fernandes BMM, Morais AM (2012) A new species of Philometra Costa, 1845 (Nematoda: Philometridae) from the freshwater fish (red piranha) Pygocentrus nattereri Kner (Characidae) in Amazonia, Brazil. Systematic Parasitolology 83: 137-144. https://doi.org/10.1007/s11230-012-9377-4

Cardia DFF, Tebaldi JH, Fornazari F, Menozzi BD, Langoni H, Nascimento AA, Bresciani KDS, Hoppe EGL (2015) Pterygodermatites (Paucipectines) andyraicola n. sp. (Spirurida: Rictulariidae), an intestinal nematode of Neotropical Molossidae bats from Brazil. Comparative Parasitology 82(2): 296-300. https://doi.org/10.1654/4748.1

Costa MAR, Maldonado Jr. A, Bóia MN, Lucio CS, Simôes RO (2014) A new species of Hassalstrongylus (Nematoda: Heligmonellidae) from Euryoryzomys russatus (Rodentia: Sigmodontinae) in the Atlantic Forest, Brazil. Neotropical Helminthology 8(2): 235-242.

De Ley P, Blaxter ML (2002) 1. Systematic position and phylogeny. In: Lee D (Ed.) The Biology of Nematodes. Harwood Academic Publishers, Reading, 1-30. https://doi.org/10.1201/ b12614-2

De Ley P, Blaxter ML (2004) A new system for Nematoda: combining morphological characters with molecular trees, and translating clades into ranks and taxa. Nematology Monographs and Perspectives 2: 633-653.

Durette-Desset MC, Gonçalves AQ, Pinto RM (2006) Trichostrongylina (Nematoda, Heligmosomoidea) coparasites in Dasyprocta fuliginosa Wagler (Rodentia, Dasyproctidae) from Brazil, with the re-establishment of the genus Avellaria Freitas \& Lent and the description of two new species. Revista Brasileira de Zoologia 23(2): 509-519. https://doi.org/10.1590/ S0101-81752006000200026

Engers KB, Boeger WA, Brandão DA (2000) Ergasilus thatcheri n. sp. (Copepoda, Poecilostomatoida, Ergasilidae) from the gills of Rhamdia quelen (Teleostei, Siluriformes, Pimelodidae) from Southern Brazil. Journal of Parasitology 86(5): 945-947. https://doi. org/10.1645/0022-3395(2000)086[0945:ETNSCP]2.0.CO;2

Feijó IA, Torres EJL, Maldonado Jr. A, Lanfredi RM (2008) A new oxyurid genus and species from Gracilinanus agilis (Marsupialia: Didelphidae) in Brazil. Journal of Parasitology 94(4): 847-851. https://doi.org/10.1645/GE-1428.1

Ferraz E, Thatcher VE (1988) Bacudacnitis grandistomis gen. et sp. nov. (Nematoda: Cucullanidae) parasita intestinal de um bagre, Pseudodoras niger (Valenciennes) da Amazonia brasileira. Amazoniana 10(3): 249-253. 
Ferraz E, Thatcher VE (1990) Camallanus acaudatus sp. n. (Nematoda, Camallanidae) and a description of the male of Camallanus tridentatus (Drasche, 1884), parasites of fishes of the Brazilian Amazon. Amazoniana 11(2): 135-141.

Ferraz E, Thatcher VE (1992) Paracamallanus amazonensis sp. n. (Nematoda: Camallanidae) um parasita de bagre, Hypophthalmus edentatus (Pisces: Hypophthalmidae) da Amazônia Brasileira. Amazoniana 12(1): 1-6.

Fiala I, Bartošová-Sojková P, Whipps CM (2015) Classification and Phylogenetics of Myxozoa. In: Okamura B, Gruhl A, Bartholomew JL (Eds) Myxozoan Evolution, Ecology and Development. Springer International Publishing, Switzerland, 85-110. https://doi. org/10.1007/978-3-319-14753-6_5

Froese R, Pauly D (2016) FishBase. World Wide Web Electronic Publication. Version 01/2016. http://www.fishbase.org [01 March 2016]

Frost DR (2016) Amphibian Species of the World: an Online Reference. Version 6.0, Electronic Database. http://research.amnh.org/herpetology/amphibia/ [14 October 2016]

García N, Morffe J (2014) A new species of Carnoya Gilson, 1898 (Rhigonematida: Carnoyidae) parasite of a spirobolid (Diplopoda: Spirobolida) from Cuba. Zootaxa 3795(5): 549556. https://doi.org/10.11646/zootaxa.3795.5.3

García N, Morffe J (2015) A new genus and two new species of Xustrostomatidae Hunt, 2002 (Nematoda: Rhigonematomorpha) from the West Indies. Papéis Avulsos de Zoologia 55(6): 91-101. https://doi.org/10.1590/0031-1049.2015.55.06

Giese EG, Lanfredi RM, Santos JN (2010) A new cucullanid species (Nematoda) from Ageneiosus ucayalensis Castelnau, 1855 (Pisces: Auchenipteridae) from Pará, Brazil. Journal of Parasitology 36(2): 389-394. https://doi.org/10.1645/GE-2081.1

Giese EG, Santos JN, Lanfredi RM (2009) A new species of Camallanidae from Ageneiosus ucayalensis (Pisces: Siluriformes) from Pará State, Brazil. Journal of Parasitology 95(2): 407-412. https://doi.org/10.1645/GE-1680.1

Gomes APN, Olifiers N, Souza JGR, Barbosa HS, D’Andrea PS, Maldonado Jr. A (2015) A new acanthocephalan species (Archiacanthocephala: Oligacanthorhynchidae) from the crab-eating fox (Cerdocyon thous) in the Brazilian Pantanal Wetlands. Journal of Parasitology 101(1): 74-79. https://doi.org/10.1645/13-321.1

Gomes DC, Lanfredi RM, Pinto RM, de Souza W (1992) Description of Trichuris travassosi n. sp. (Nematoda: Trichurinae) from a Brazilian rodent, by light and scanning electron microscopy. Memórias do Instituto Oswaldo Cruz 87(1): 1-10. https://doi.org/10.1590/ S0074-02761992000500004

Gonçalves AQ, Pinto RM, Durette-Desset M (2007) Parasitism of two zoonotic reservoirs Dasyprocta leporina and D. fuliginosa (Rodentia) from Amazonas, with Trichostrongylina nematodes (Heligmonellidae): description of a new genus and a new species. Memórias do Instituto Oswaldo Cruz 102(6): 763-768. https://doi.org/10.1590/S007402762007000600017

Hoppe EGL, Araújo RCL, Tebaldi JH, Nascimento AA (2010) Pterygodermatites (Multipectines) pluripectinata n. sp. (Spirurida: Rictulariidae), a nematode parasite of the crab-eating fox Cerdocyon thous (Linnaeus, 1766) from Caatinga shrubland, Brazil. Journal of Helminthology 84(3): 312-316. https://doi.org/10.1017/S0022149X0999071X 
Hoppe EGL, Nascimento AA (2007) Natural infection of gastrointestinal nematodes in longnosed armadillos Dasypus novemcinctus Linnaeus, 1758 from Pantanal wetlands, Aquidauana sub-region, Mato Grosso do Sul State, with the description of Hadrostrongylus speciosum n. gen. et n. sp. (Molineidae: Anoplostrongylinae). Veterinary Parasitology 144(1-2): 87-92. https://doi.org/10.1016/j.vetpar.2006.09.028

ICZN (International Commission on Zoological Nomenclature) (1999) International code of zoological nomenclature. Fourth Edition. The International Trust for Zoological Nomenclature, London.

Jiménez-Ruiz FA, Gardner SL (2003) The nematode fauna of long-nosed mice Oxymycterus spp. from the Bolivian yungas. Journal of Parasitology 89(2): 299-308. https://doi. org/10.1645/0022-3395(2003)089[0299:TNFOLM]2.0.CO;2

Jiménez-Ruiz FA, Gardner SL, Varela-Stokes AS (2006) Aspidoderidae from North America, with the description of a new species of Aspidodera (Nematoda: Heterakoidea). Journal Parasitology 92(4): 847-854. https://doi.org/10.1645/GE-735R.1

Knoff M, Felizardo NN, Ińiguez AM, Maldonado Jr. A, Torres EJL, Pinto RM, Gomes DC (2012) Genetic and morphological characterisation of a new species of the genus Hysterothylacium (Nematoda) from Paralichthys isosceles Jordan, 1890 (Pisces: Teleostei) of the Neotropical Region, state of Rio de Janeiro, Brazil. Memórias do Instituto Oswaldo Cruz 107(2): 186-193. https://doi.org/10.1590/S0074-02762012000200006

Knoff M, Noronha D, Magalhães-Pinto R, Sanches M, Siqueira LR, Mattos FB, Gomes DC (2010) Coleção Helminológica do Instituto Oswaldo Cruz (CHIOC). In: Lamothe-Argumedo R, Damborenea C, García-Prieto L, Lunaschi LI, Osorio-Sarabia D (Comps) Guide to Helminthological Collections of Latin America. Publicaciones Especiales 22, Instituto de Biología, Universidad Nacional Autónoma de México and Museo de La Plata, Universidad Nacional de La Plata, Buenos Aires and Mexico City, 15-27.

Kohn A, Fernandes BMM (1988a) Helminth parasites of fishes from the hydroeletric power station of Eletrosul (Brazil). I: Procamallanus petterae n. sp. and Spirocamallanus pintoi n. sp. (Nematoda, Camallanidae) from the reservoir of "Salto Osório". Memórias do Instituto Oswaldo Cruz 83(3): 293-298. https://doi.org/10.1590/S007402761988000300005

Kohn A, Fernandes BMM (1988b) Procamallanus annipetterae nom. nov. for Procamallanus petterae Kohn \& Fernandes, 1988 preocupied by Procamallanus (Procamallanus) petterae Moravec \& Sey, 1988. Memórias do Instituto Oswaldo Cruz 83(4): 535. https://doi. org/10.1590/S0074-02761988000400025

Lacerda ACF, Takemoto RM, Marchiori NC, Martins ML, Pavanelli GC (2013) New species of Cucullanus (Nematoda: Cucullanidae), an intestinal parasite of the peacock bass Cichla piquiti (Perciformes: Cichlidae) from the Tocantins River, Brazil. Journal of Helminthology: 1-4. https://doi.org/10.1017/S0022149X13000485

Lent H, Santos CP (1989) Acanthocephalus ula n. sp. (Acanthocephala, Echinorhynchidae) and Schulzia usu n. sp. (Nematoda, Molineidae) described from Atelopus oxyrhynchus (Amphibia) from Venezuela. Revista Brasileira de Biologia 49(4): 1085-1091.

Lepage J, Warnier J (2014) The Peters' Check-list of the Birds of the World (1931-1987) Database. http://avibase.bsc-eoc.org/peterschecklist.jsp [14 October 2016] 
Lopes DA, Mainenti A, Knoff M, Gomes DC (2017) Type material of Platyhelminthes housed in the Helminthological Collection of the Oswaldo Cruz Institute/ FIOCRUZ (CHIOC), Rio de Janeiro, Brazil, from 1979 to 2016 (Rhabditophora, Trematoda and Cestoda). ZooKeys 662: 1-48. https://doi.org/10.3897/zookeys.662.11685

Lopes DA, Mainenti A, Sanches M, Knoff M, Gomes DC (2016) Type material of Platyhelminthes (Monogenoidea) housed in the Helminthological Collection of the Oswaldo Cruz Institute/ FIOCRUZ (CHIOC), Rio de Janeiro, Brazil, from 1979 to 2016 . ZooKeys 616: 1-75. https://doi.org/10.3897/zookeys.616.8481

Lopes Torres EJ, Maldonado Jr. A, Lanfredi RM (2009) Spirurids from Gracilinanus agilis (Marsupialia: Didelphidae) in Brazilian Pantanal wetlands with a new species of Physaloptera (Nematoda: Spirurida). Veterinary Parasitology 163(1-2): 87-92. https://doi. org/10.1016/j.vetpar.2009.03.046

Lopes Torres EJ, Nascimento APF, Menezes AO, Garcia J, Santos MAJ, Maldonado Jr. A, Miranda K, Lanfredi RM, Souza W (2011) A new species of Trichuris from Thrichomys apereoides (Rodentia: Echimyidae) in Brazil: morphological and histological studies. Veterinary Parasitology 176: 226-235. https://doi.org/10.1016/j.vetpar.2010.10.053

Luque JL, Alves DR (2003) Pseudolernentoma brasiliensis n. g., n. sp. (Copepoda: Poecilostomatoida: Chondracanthidae) parasitic on Genypterus brasiliensis (Osteichthyes: Ophidiidae) from off the State of Rio de Janeiro, Brazil. Systematic Parasitolology 56(3): 195-199. https://doi.org/10.1023/B:SYPA.0000003806.30680.1b

Martin JW, Davis GE (2001) An updated classification of the recent Crustacea. Natural History Museum of Los Angeles County Science Series 39(1): 1-124.

Martins ML, Garcia F, Piazza RS, Ghiraldelli L (2007) Camallanus maculatus n. sp. (Nematoda: Camallanidae) in an ornamental fish Xiphophorus maculatus (Osteichthyes: Poeciliidae) cultivated in São Paulo State, Brazil. Arquivo Brasileiro de Medicina Veterinária e Zootecnia 59(5): 1224-1230. https://doi.org/10.1590/S0102-09352007000500019

Martins ML, Onaka E (2006) Henneguya garavelli n. sp. and Myxobolus peculiaris n. sp. (Myxozoa: Myxobolidae) in the gills of Cyphocharax nagelli (Osteichthyes: Curimatidae) from Rio do Peixe Reservoir, São José do Rio Pardo, São Paulo, Brazil. Veterinary Parasitology 137: 253-261. https://doi.org/10.1016/j.vetpar.2005.12.023

Martins ML, Yoshitoshi ER (2003) A new nematode species Goezia leporini n. sp. (Anisakidae) from cultured freshwater fish Leporinus macrocephalus (Anostomidae) in Brazil. Brazilian Journal of Biology 63(3): 497-506. https://doi.org/10.1590/S1519-69842003000300016

Martins ML, Yoshitoshi ER, Umekita H (2001) Ichthyouris voltagrandensis n. sp. (Nematoda: Pharyngodonidae) from Myleus tiete Eigenmann \& Norris, 1900 (Osteichthyes: Characidae) in the Volta Grande Reservoir, MG, Brazil. Revista Brasileira de Biologia 61(2): 305310. https://doi.org/10.1590/S0034-71082001000200013

Melo MFC, Santos JN, Giese EG, Santos EGN, Santos CP (2011) Raphidascaris (Sprentascaris) lanfrediae sp. nov. (Nematoda: Anisakidae) from the fish Satanoperca jurupari (Osteichthyes: Cichlidae). Memórias do Instituto Oswaldo Cruz 106(5): 553-556. https://doi. org/10.1590/S0074-02762011000500006

Monteiro CM, Amato JFR, Amato SB (2006) A new species of Andracantha Schmidt (Acanthocephala, Polymorphidae) parasite of Neotropical cormorants, Phalacrocorax brasilianus 
(Gmelin) (Aves, Phalacrocoracidae) from southern Brazil. Revista Brasileira de Zoologia 23(3): 807-812. https://doi.org/10.1590/S0101-81752006000300027

Moraes Neto AHA, Lanfredi RM, Souza W (1997) Litomosoides chagasfilhoi sp. nov. (Nematoda: Filarioidea) parasitizing the abdominal cavity of Akodon cursor (Winge, 1887) (Rodentia: Muridae) from Brazil. Parasitology Research 83(2): 137-143. https://doi.org/10.1007/ s004360050223

Morais DH, Aguiar A, Müller MI, Narciso RB, Silva LAF, Silva RJ (2016). Morphometric and phylogenetic analyses of the Serpentirhabdias viperidicus n. sp. (Nematoda: Rhabdiasidae) from the lancehead snake Bothrops moojeni Hoge, 1966 (Reptilia: Serpentes: Viperidae) in Brazil. Journal of Helminthology: 1-11. https://doi.org/10.1017/S0022149X16000377

Morales-Serna FN, Gómez S (2010) A new bomolochid copepod parasitic on bullseye puffer Sphoeroides annulatus (Jenyns) from Mexico, with reassignment of some species of Acantholochus Cressey and Hamaticolax Ho \& Lin. Zootaxa 2336: 36-50.

Moravec F, Kohn A, Fernandes BMM (1992a) Three new species of oxyuroid nematodes, including two new genera, from freshwater catfishes in Brazil. Systematic Parasitology 21(3): 189-201. https://doi.org/10.1007/BF00009699

Moravec F, Kohn A, Fernandes BMM (1992b) Nematode parasites of fishes of the Parana river, Brazil. 1. Trichuroidea, Oxyuroidea and Cosmocercoidea. Folia Parasitologica 39(4): 327-353.

Moravec F, Kohn A, Fernandes BMM (1992c) Neoparaseuratum travassosi n. g., n. sp. (Nematoda: Quimperiidae), a new parasite from thorny catfish Pterododas granulosus in Brazil. Memórias do Instituto Oswaldo Cruz 87(1): 145-150. https://doi.org/10.1590/S007402761992000500029

Moravec F, Kohn A, Fernandes BMM (1993) Nematode parasites of fishes of the Parana river, Brazil. 2. Seuratoidea, Ascaridoidea, Habronematoidea and Acuarioidea. Folia Parasitologica 40(2): 115-134.

Moravec F, Kohn A, Fernandes BMM (1994) 2. New species of the genus Goezia, G. brasiliensis sp n and G. brevicaeca sp n (Nematoda, Anisakidae), from fresh-water fishes in Brazil. Folia Parasitologica 41(4): 271-278.

Moravec F, Kohn A, Fernandes BMM (1997) New observations on seuratoid nematodes parasitic in fishes of the Paraná River, Brasil. Folia Parasitologica 44(3): 209-223.

Moravec F, Santos CP (2009) Dracunculus brasiliensis sp. n. (Nematoda: Dracunculidae) from the anaconda, Eunectes murinus (Ophidia: Boidae). Parasitology Research 104: 589-592. https://doi.org/10.1007/s00436-008-1234-7

Moreira NIB, Rocha GN, Costa HMA (2000) A new nematode species (Seuratoidea, Cucullanidae) parasitizing Parauchenipterus striatulus (Steindachner, 1876) (Pisces, Auchenipteridae) in Brazil. Memórias do Instituto Oswaldo Cruz 95(1): 39-41. https://doi. org/10.1590/S0074-02762000000100005

Morffe J, García N (2010a) A new specie of Artigasia Christie, 1934 (Oxyurida: Hystrignathidae) from a Cuban passalid beetle. Papéis Avulsos de Zoologia 50(36): 571-577. https:// doi.org/10.1590/S0031-10492010003600001

Morffe J, García N (2010b) Hystrignathus dearmasi sp. n. (Oxyurida, Hystrignathidae), first record of a nematode parasitizing a Panamanian Passalidae (Insecta, Coleoptera). ZooKeys 57: 1-8. https://doi.org/10.3897/zookeys.57.477 
Morffe J, García N (2013a) Batwanema gen. n. and Chokwenema gen. n. (Oxyurida, Hystrignathidae), new nematode genera as parasites of Passalidae (Coleoptera) from the Democratic Republic of Congo. ZooKeys 361: 1-13. https://doi.org/10.3897/zookeys.361.6351

Morffe J, García N (2013b) Two new genera of nematode (Oxyurida, Hystrignathidae) parasites of Passalidae (Coleoptera) from the Democratic Republic of Congo. ZooKeys 257: 1-15. https://doi.org/10.3897/zookeys.257.3666

Noronha D (1992) On Machadosentis travassosi n. g., n. sp. (Eoacanthocephala: Quadrigyridae) parasite of a moray Gymnothorax ocellatus in Brazil. Memórias do Instituto Oswaldo Cruz 87(1): 151-153. https://doi.org/10.1590/S0074-02761992000500030

Panizzutti MHM, Santos LC, Vicente JJ, Muniz-Pereira LC, Pinto RM (2003) Ophidascaris durissus sp. nov. (Nematoda Ascarididae) parasitizing Crotalus durissus Linnaeus (Ophidia, Viperidae) in Brazil. Revista Brasileira de Zoologia 20(1): 9-11. https://doi.org/10.1590/ S0101-81752003000100003

Paschoal F, Vieira FM, Cezar AD, Luque JL (2014) Dichelyne (Cucullanellus) tornquisti n. sp. (Nematoda: Cucullanidae) from corocoro grunt, Orthopristis ruber (Cuvier, 1830) (Perciformes: Haemulidae) from Southeastern Brazil. Journal of Parasitology 100(2): 215-220. https://doi.org/10.1645/13-334.1

Peralta ASL, Matos ER, Serra-Freire NM (1998) Unoculubranchiobdella expansa gen. nov., sp. n. (Hirudinea: Ozobranchidae) parasito de Podocnemis expansa (Chelonia: Pelomedusidae). Entomologia y Vectores 5(5): 161-177.

Pereira AN, Timi JT, Vieira FM, Luque JL (2012a) A new species of Neoascarophis (Nematoda: Cystidicolidae) parasitic in Mullus argentinae (Perciformes: Mullidae) from the Atlantic coast of South America. Folia Parasitologica 59(1): 64-70. https://doi.org/10.14411/fp.2012.010

Pereira FB, Alves PV, Rocha BM, Lima SS, Luque JL (2012b) A new Physaloptera (Nematoda: Physalopteridae) parasite of Tupinambis merianae (Squamata: Teiidae) from southeastern Brazil. Journal of Parasitology 98(6): 1227-1235. https://doi.org/10.1645/GE-3159.1

Pereira FB, Alves PV, Rocha BM, Lima SS, Luque JL (2014a) Physaloptera bainae n. sp. (Nematoda: Physalopteridae) parasitic in Salvator merianae (Squamata: Teiidae), with a key to Physaloptera species parasitizing reptiles from Brazil. Journal of Parasitology 100(2): 221227. https://doi.org/10.1645/13-281.1

Pereira FB, Pereira AN, Luque JL (2014b) A new species of Comephoronema (Nematoda: Cystidicolidae) from the squirrelfish Holocentrus adscensionis (Beryciformes: Holocentridae) off Brazil. Folia Parasitologica 61(1): 55-62. https://doi.org/10.14411/fp.2014.001

Pereira FB, Pereira AN, Timi JT, Luque JL (2013) Pseudascarophis brasiliensis sp. nov. (Nematoda: Cystidicolidae) parasitic in the Bermuda chub Kyphosus sectatrix (Perciformes: Kyphosidae) from southeastern Brazil. Memórias do Instituto Oswaldo Cruz 108(4): 476-480. https://doi.org/10.1590/S0074-0276108042013013

Pereira FB, Tavares LER, Scholz T, Luque JL (2015) A morphological and molecular study of two species of Raphidascaroides Yamaguti, 1941 (Nematoda: Anisakidae), parasites of doradid catfish (Siluriformes) in South America, with a description of $R$. moraveci n. sp. Systematic Parasitology 91(1): 49-61. https://doi.org/10.1007/s11230-015-9555-2

Pereira FB, Vieira FM, Luque JL (2014c) A new species of Cucullanus Müller, 1777 (Nematoda: Cucullanidae) parasitic in the grey triggerfish Balistes capriscus Gmelin (Osteichthyes: 
Balistidae) off Rio de Janeiro, Brazil. Systematic Parasitology 87(3): 283-291. https://doi. org/10.1007/s11230-014-9470-y

Pereira Jr. J, da Costa MAS (1996) Cucullanidae (Nematoda: Seuratoidea) em Micropogonias furnieri (Desmarest, 1823) (Sciaenidae) do Rio Grande do Sul, com a descrição de Cucullanus cassinensis sp. n. e Dichelyne (Dichelyne) micropogonii sp. n. Comunicaçóes do Museu de Ciências da PUCRS, Porto Alegre 9: 11-30.

Perez-Asso AR (1996) The genus Nesobolus (Diplopoda: Spirobolida: Rhinocricidae) in Cuba. Insecta Mundi 10(1-4): 1-11.

Pinto RM, Gomes DC (1985) Nematodes of Amazonian birds with a description of Hoazinstrongylus amazonensis n. gen. n. sp. (Trichostrongylidae, Libyostrongylinae). Memórias do Instituto Oswaldo Cruz 80(2): 213-217. https://doi.org/10.1590/S0074-02761985000200012

Pinto RM, Kohn A, Fernandes BMM, Mello DA (1982) Nematodes of rodents in Brazil, with description of Aspidodera vicentei n.sp. Systematic Parasitology 4(3): 263-267. https://doi. org/10.1007/BF00009627

Pinto RM, Vicente JJ (1995) Tetrameres (Tetrameres) spirospiculum n. sp. (Nematoda, Tetrameridae) from the buff-necked Ibis, Theristicus caudatus caudatus (Boddaert) (Aves, Threskiornithidae). Memórias do Instituto Oswaldo Cruz 90(5): 615-617. https://doi.org/10.1590/ S0074-02761995000500013

Pinto RM, Vicente JJ, Noronha D (1981) Diplotriaena zederi sp. n. (Diplotriaenidae, Filarioidea) from wood hewer (Xiphocolaptes albicollis albicollis) in Brazil. Atas da Sociedade de Biologia do Rio de Janeiro 22: 37-38.

Pinto RM, Vicente JJ, Noronha D (1984) First report of Ascarophis van Beneden, 1871: A. brasiliensis n. sp. (Nematoda, Ascarophidinae) and Procamallanus (Spirocamallanus) pereirai Annereaux, 1946 (Nematoda, Procamallaninae) in South America. Memórias do Instituto Oswaldo Cruz 79(4): 491-494. https://doi.org/10.1590/S0074-02761984000400015

Pinto RM, Vicente JJ, Noronha D (1996) Nematode parasites of Brazilian piciformes birds: a general survey with description of Procyrnea anterovulvata n. sp. (Habronematoidea, Habronematidae). Memórias do Instituto Oswaldo Cruz 91(4): 479-487. https://doi. org/10.1590/S0074-02761996000400015

Rego AA (1982) Helminthological Collection of the Oswaldo Cruz Institute. In: Lichtenfels JR, Pritchard MH (Eds) A Guide to Parasite Collection of the World. American Society of Parasitologists, Special Publications, Lawrence, 79 pp.

Rego AA, Vicente JJ, Gomes DC (1979) List of holotypes in the Helminthological Collection of the Oswaldo Cruz Institute. Atas da Sociedade de Biologia do Rio de Janeiro 19 (Suppl): 1-29.

Rodrigues HO (1992) Pseudocapillaria (Ichthyocapillaria) maricaensis n. sp. (Nematoda, Capillariidae) and remarks on the helminthological fauna of Liolaemus lutzae Mertens, 1938 (Lacertilia, Iguanidae). Memórias do Instituto Oswaldo Cruz 87(2): 297-300. https://doi. org/10.1590/S0074-02761992000200019

Rodrigues HO, Rodrigues SS (1980) Nova espécie do gênero Aprocta Linstow, 1883 (Nematoda, Aproctoidea). Atas da Sociedade de Biologia do Rio de Janeiro 21: 12-14.

Rodrigues HO, Vicente JJ, Gomes DC (1985) Strongyloides ferrerai sp. n. (Nematoda, Rhabdiasoidea) parasito do roedor Kerodon rupestris (Wied.) no Brasil. Memórias do Instituto Oswaldo Cruz 80(4): 407-410. https://doi.org/10.1590/S0074-02761985000400005 
Salgado-Maldonado G, Santos CP (2000). Amapacanthus amazonicus n. g., n. sp. (Acanthocephala: Diplosentidae: Allorhadinorhynchinae) from Arius passany and Anableps microleps (Pisces) at Maraca Island off northern Brazil. Systematic Parasitology 46(2): 111-116. https://doi.org/10.1023/A:1006327308667

Santos CP, Lent H, Gibson DI (2004) A new species of Pseudanisakis Layman \& Borovkova, 1926 (Nematoda: Ascaridida) from Rioraja agassizii and Psammobatis extenta (Rajidae) in Brazilian southwestern Atlantic waters. Systematic Parasitology 57(3): 229-235. https:// doi.org/10.1023/B:SYPA.0000019086.44939.17

Santos CP, Moravec F, Venturieri R (2008a) Capillostrongyloides arapaimae sp. n. (Nematoda: Capillariidae), a new intestinal parasite of the arapaima Arapaima gigas from the Brazilian Amazon. Memórias do Instituto Oswaldo Cruz 103(4): 392-395. https://doi.org/10.1590/ S0074-02762008000400013

Santos JN, Giese EG, Maldonado Jr. A, Lanfredi RM (2008b) A new species of Oswaldocruzia (Molineidae: Nematoda) in Chaunus marinus (Amphibian: Bufonidae) (Linnaeus, 1758) from Brazil. Journal of Parasitology 94(1): 264-268. https://doi.org/10.1645/GE-1336.1

Santos JN, Melo FTV, Nascimento LCS, Nascimento DEB, Giese EG, Furtado AP (2011) Rhabdias paraensis sp. nov.: a parasite of the lungs of Rhinella marina (Amphibia: Bufonidae) from Brazilian Amazonia. Memórias do Instituto Oswaldo Cruz 106(4): 433-440. https://doi.org/10.1590/S0074-02762011000400008

Santos MD, Thatcher VE, Brasil-Sato MC (2007) Brasergasilus bifurcatus sp. nov. (Copepoda, Ergasilidae, Abergasilinae) from the gills and nasal fossae of serrasalmid fishes from the Três Marias Reservoir, Upper São Francisco River, Minas Gerais State, Brazil. Acta Parasitologica 52(3): 268-272. https://doi.org/10.2478/s11686-007-0036-x

São Luiz J, Simóes RO, Lopes Torres E, Barbosa HS, Santos JN, Giese EG, Rocha FL, Maldonado Jr. A (2015) A new species of Physaloptera (Nematoda: Physalopteridae) from Cerradomys subflavus (Rodentia: Sigmodontinae) in the Cerrado Biome, Brazil. Neotropical Helminthology 9(2): 301-312.

Sawyer RT (1986) Leech Biology and Behavior. Clarendon Press, Oxford, 1065 pp.

Simóes RO, Santos MM, Maldonado Jr. A (2012) A new Heligmonellid (Nematoda: Heligmonellidae) from Oecomys mamorae (Rodentia: Sigmodontinae) in the Pantanal and new data on the synlophe of Guerrerostrongylus zetta from the Atlantic forest, Brazil. Journal of Parasitology 98(4): 801-805. https://doi.org/10.1645/GE-2905.1

Siqueira LR, Panizzutti MHM, Muniz-Pereira LC, Pinto RM (2005) Description of a new ascaridoid parasite of Bothrops jararaca Wied (Reptilia, Ophidia) in Brazil. Revista Brasileira de Zoologia 22(3): 587-590. https://doi.org/10.1590/S0101-81752005000300010

Souza JGR, Digiani MC, Simôes RO, Luque JL, Rodrigues-Silva R, Maldonado Jr. A (2009) A new heligmonellid species (Nematoda) from Oligoryzomys nigripes (Rodentia: Sigmodontinae) in the Atlantic Forest, Brazil. Journal of Parasitology 95(3): 734-738. https://doi. org/10.1645/GE-1836.1

Tavares LER, Luque JL (2003) A new species of Acantholochus (Copepoda: Bomolochidae) parasitic on Centropomus undecimalis (Osteichthyes: Centropomidae) from the coastal zone of the State of Rio de Janeiro, Brazil. Memórias do Insituto Oswaldo Cruz 98(2): 241-245. https://doi.org/10.1590/S0074-02762003000200013 
Tavares LER, Luque JL (2005) Ergasilus youngi sp. nov. (Copepoda, Poecilostomatoida, Ergasilidae) parasitic on Aspistor luniscutis (Actinopterygii, Ariidae) from off the State of Rio de Janeiro, Brazil. Acta Parasitologica 50(2): 150-155.

Timi JT, Lanfranchi AL, Tavares LER, Luque JL (2009) A new species of Dichelyne (Nematoda, Cucullanidae) parasitizing sciaenid fishes from off South American Atlantic coast. Acta Parasitologica 54(1): 45-52. https://doi.org/10.2478/s11686-009-0010-x

Uetz P, Freed P, Hošek J (2016) The Reptile Database. http://www.reptile-database.org [14 October 2016]

Vicente JJ, Pinto RM, Noronha D (1980) Sobre um novo gênero e duas novas espécies de nematódeos parasitas de aves brasileiras. Atas da Sociedade de Biologia do Rio de Janeiro 21: 75-79.

Vicente JJ, Pinto RM, Noronha D (1993) Remarks, on six species of heterakid nematodes parasites of brazilian tinamid birds with description of a new species. Memórias do Instituto Oswaldo Cruz 88(2): 271-278. https://doi.org/10.1590/S0074-02761993000200015

Vicente JJ, Pinto RM, Noronha D (1996) Synhimantus (Synhimantus) magnipapillatus n. sp. (Nematoda, Acuarioidea) from the yellow-crowned night-heron, Nyctanassa violacea cayennensis (Gmelin) (Aves, Ardeidae). Memórias do Instituto Oswaldo Cruz 91(1): 51-53. https://doi.org/10.1590/S0074-02761996000100008

Vicente JJ, Van Sluys M, Fontes AF, Kiefer MC (2000a) Subulura lacertilia sp.n. (Nematoda, Subuluridae) parasitizing the Brazilian lizard Tropidurus nanuzae Rodrigues (Lacertilia, Tropiduridae). Revista Brasileira de Zoologia 17(4): 1065-1068. https://doi.org/10.1590/ S0101-81752000000400013

Vicente JJ, Vrcibradic D, Muniz-Pereira LC, Pinto RM (2000b) Skrjabinodon heliocostai sp. n. (Nematoda, Pharyngodonidae) parasitizing Mabuya frenata (Cope) (Lacertilia, Scincidae) in Brazil and the reallocation of Skrjabinodon capacyupanquii (Freitas, Vicente \& Ibanez) in the genus Thelandros Wedl. Revista Brasileira de Zoologia 17(2): 361-367. https://doi. org/10.1590/S0101-81752000000200006

Vicente JJ, Vrcibradic D, Rocha CFD, Pinto RM (2002) Description of Skrjabinodon spinosulus sp. n. (Nematoda, Oxyuroidea, Pharyngodonidae) from the Brazilian lizard Mabuya dorsivittata Cope, 1862 (Scincidae). Revista Brasileira de Zoologia 19(1): 157-162. https://doi. org/10.1590/S0101-81752002000100014

Vieira FM, Felizardo NN, Luque JL (2009) A new species of Heterosentis Van Cleave, 1931 (Acanthocephala: Arhythmacanthidae) parasitic in Pseudopercis numida Miranda Ribeiro, 1903 (Perciformes: Pinguipedidae) from Southeastern Brazilian coastal zone. Journal of Parasitology 95(3): 747-750. https://doi.org/10.1645/GE-1855.1

Vieira FM, Muniz-Pereira LC, Lima SS, Moraes Neto AHA, Gonçalves PR, Luque JL (2012). Crenosoma brasiliense sp. n. (Nematoda: Metastrongyloidea) parasitic in lesser grison, Galictis cuja (Molina, 1782) (Carnivora, Mustelidae) from Brazil, with a key to species of Crenosoma Molin, 1861. Folia Parasitologica 59(3): 187-194. https://doi.org/10.14411/fp.2012.026

Vieira FM, Muniz-Pereira LC, Lima SS, Moraes Neto AHA, Guimarães EV, Luque JL (2013) A new metastrongyloidean species (Nematoda) parasitizing pulmonary arteries of Puma (Herpailurus) yagouaroundi (E. Geoffroy, 1803) (Carnivora: Felidae) from Brazil. Journal of Parasitology 99(2): 327-331. https://doi.org/10.1645/GE-3171.1 
Vieira FM, Pereira FB, Pantoja C, Soares IA, Pereira AN, Timi JT, Scholz T, Luque JL (2015) A survey of nematodes of the genus Cucullanus Müller, 1777 (Nematoda, Seuratoidea) parasitic in marine fishes off Brazil, including description of three new species. Zootaxa 4039(2): 289-311. https://doi.org/10.11646/zootaxa.4039.2.5

Weirich JM, Catzeflis F, Jiménez FA (2016) Guerrerostrongylus marginalis n. sp. (Trichostrongyloidea: Heligmonellidae) from the Guianan arboreal mouse (Oecomys auyantepui) from French Guiana. Parasite 23(9): 1-10. https://doi.org/10.1051/parasite/2016009

Wilson DO, Reeder DM (2005) Mammal species of the world, 3rd Edition. http://vertebrates. si.edu/msw/mswcfapp/msw [14 October 2016]

Young PS (1998) Catalogue of Crustacea of Brazil. Rio de Janeiro: Museu Nacional, Série Livros 6, 717. 